\title{
The Lattice Boltzmann Method for Flapping Wing Aerodynamics
}

\author{
Hikaru Aono ${ }^{1, a}$, Amit Gupta ${ }^{2, a}$, Dewei Qi ${ }^{3, b}$ and Wei Shyy ${ }^{4, a}$ \\ ${ }^{a}$ University of Michigan, Ann Arbor, Michigan, 48109, USA \\ ${ }^{b}$ Western Michigan University, Kalamazoo, Michigan, 49008, USA
}

\begin{abstract}
The lattice Boltzmann method offers an alternative framework compared to the NavierStokes simulations. However, the applicability of this method to simulate moving boundary problems has not received sufficient attention. In this work, different treatments of the noslip condition of lattice Boltzmann method for the moving boundary problem of rigid and flexible wings flapping in a fluid have been analyzed. Schemes based on interpolation have been considered in the scope of flapping and plunging wing simulations to analyze the accuracy of the solution near the moving boundary. It is shown that interpolation resolves the exact location of the wall accurately when the distance from the nearest lattice, $\Delta \neq 1 / 2$, unlike the halfway bounceback method. Flow past a vertically oscillating plate at $\operatorname{Re}=\mathbf{1 0 0}$ with two different amplitudes is simulated. The presence of large fluctuations in forces, due to different levels of accuracy of pressure and velocity, at the time instant when an oscillating plate crosses into the adjoining lattice is shown. For a three-dimensional zerothickness flat plate undergoing hovering motion with delayed rotation, interpolation schemes show better agreement with Navier-Stokes solution as compared to the halfway bounceback method. The flow field computed using interpolation indicates a continuous variation of pressure and vorticity near the surface of the wing. Lattice Boltzmann simulation of a finitethickness flat plate is also shown to be in good agreement with the Navier-Stokes solution. Preliminary results of a two-link flexible wing model undergoing pitching and plunging motion are also presented. Through simulations using the flexible-particle model, good agreement with the force and deflection angle recorded in earlier experiments is reported.
\end{abstract}

\section{Nomenclature}

$\mathbf{A}_{i} \quad=$ transformation matrix from the space coordinate to the body-fixed coordinate

$\mathrm{b}=$ wing span

$C_{D} \quad=$ drag coefficient

$C_{L} \quad=$ lift coefficient

$c=$ chord length of flat plate

$c_{S} \quad=$ speed of sound

$\mathbf{e}_{\alpha} \quad=$ phase space velocity vector

$\mathbf{e}_{\bar{\alpha}} \quad=$ phase space velocity vector opposite to $\mathbf{e}_{\alpha}$

$E \quad=$ Young's modulus of the segment

$f \quad=$ excitation frequency of the wing

$f_{\alpha} \quad=$ distribution function

$\mathbf{F}_{i}^{h} \quad=$ hydrodynamic forces acting on segment $i$

$G \quad=$ Shear modulus of segment $i$

$G_{r}, G_{t}=$ rotational and translational shape functions

\footnotetext{
${ }^{1}$ Postdoctoral research fellow, Department of Aerospace Engineering, aonoh@umich.edu, AIAA member.

${ }^{2}$ Postdoctoral research fellow, Department of Aerospace Engineering, amitgupt@umich.edu, AIAA member.

${ }^{3}$ Professor, Department of Paper Engineering, Chemical Engineering and Imaging, dewei.qi@.wmich.edu, AIAA member.

${ }^{4}$ Clarence L. 'Kelly' Johnson Collegiate Professor and Chairman, Department of Aerospace Engineering, weishyy@umich.edu, AIAA fellow.
} 


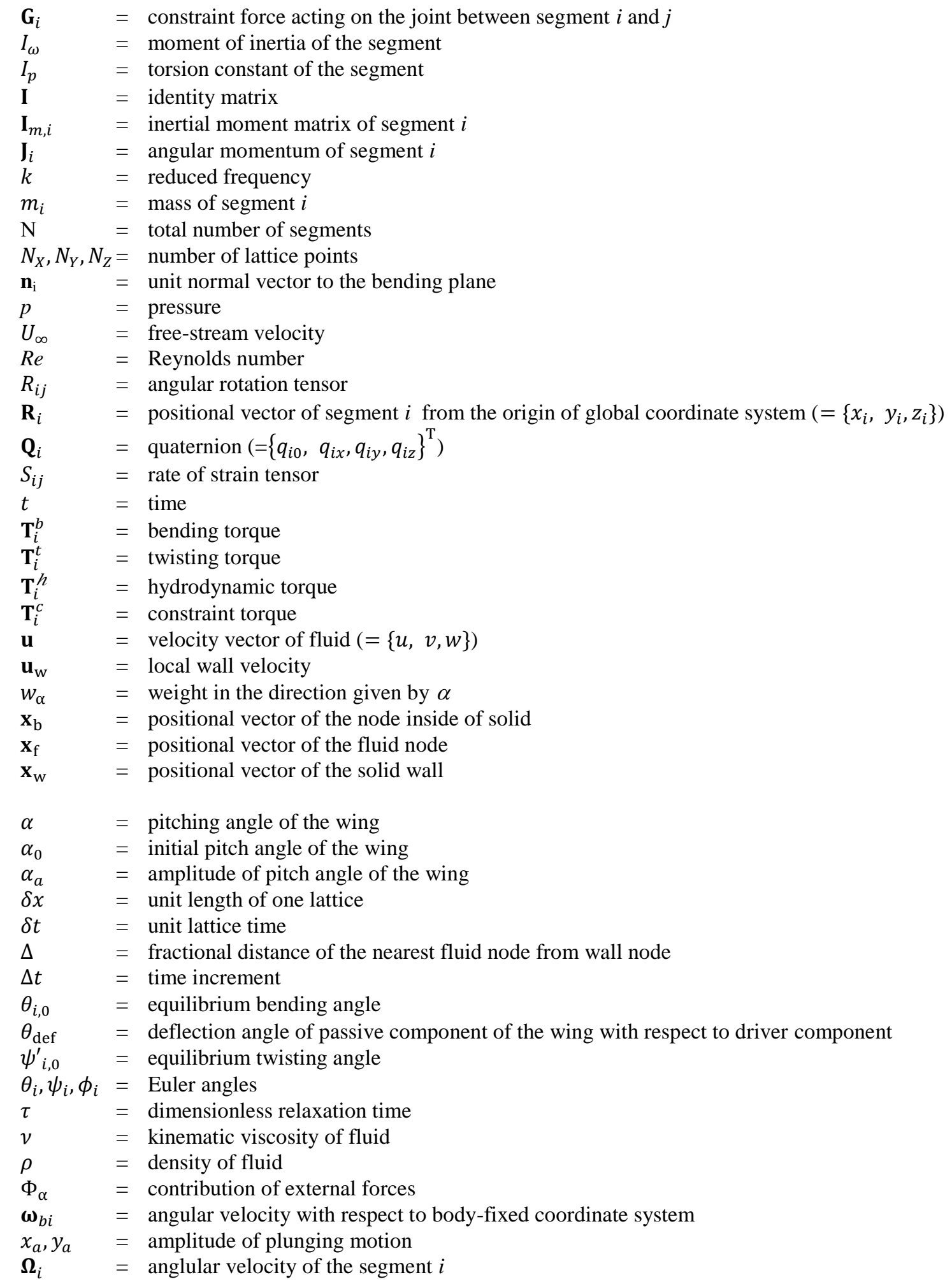

\section{Introduction}

MICRO air vehicles (MAVs) have generated substantial and rapidly growing interest in the engineering and science communities. The MAVs are originally defined as flying vehicle about $15 \mathrm{~cm}$ in size and are 
developed to reconnoiter in confined spaces (inside buildings, tunnels etc). This requires power-efficiency, highmaneuverability, and low-speed flight with stable hover. With the rapid progress made in structural and material technologies, miniaturization of power plants, communication, visualization, and control devices, numerous MAVs have been developed. Overall, alternative MAV concepts, based on fixed wing, rotary wing, and flapping wing have been investigated [1-3]. One of most successful fixed-winged MAV's size is just barely over $10 \mathrm{~cm}$ [4].

MAVs usually operate in the low Reynolds number regime (less than $O\left(10^{5}\right)$ ), which compared with large, manned flight vehicles have unfavorable aerodynamic performances, such as low lift-to-drag ratio. On the other hand, the MAV's small geometric dimension results in favorable scaling characteristics, such as reduced stall speed and better structural survivability. In the flapping-wing MAV development, there is great potential for collaborative research between biologists and engineers as MAVs and biological flyers share similar dimensions, weight, flight speeds, and flight environment. With respect to maneuvering a body efficiently through space, birds not only represent some of nature's finest locomotion experiments but also show greater performance than human-made aircrafts. The primary reasons for such superior maneuvering and flight characteristics include the 'scaling laws' with respect to a vehicle's size and material property, as well as intuitive but well-developed sensing, navigation, and control capabilities [1,5]. There are general references that offer broad accounts of biological flight including geometric scaling laws, power, morphology, simplified modeling, and unsteady aerodynamics [1].

Numerous studies have shown that biological flapping flyers utilize several unsteady mechanisms to keep afloat and control their flight [1,5-12]. However, the role of these mechanisms is dependent on wing kinematics, wing deformation, size of flyer and flight environment. Specifically, enhancement of aerodynamic force due to leading edge vortex (LEV) generation is the most likely general mechanism in flapping flight [1, 5, 9-12]. In addition to the LEV, wake-capturing mechanism is sensitive to wake structure and orientation of wing when the two interact [5], and tip vortices can enhance lift generation when the proper wing kinematics is utilized [6]. Some other unsteady aerodynamic mechanisms that could play a role are discussed in literature as well [1,5].

Numerical simulations performed to investigate fluid physics that emanates from the flapping motion of a wing have primarily relied on employing the Navier-Stokes equation-based macroscopic method. These approaches have been very successful to solve flow field around moving boundaries. However, considerable challenges still exist, such as the development of robust and computationally efficient schemes of remeshing, grid generation, and efficient matrix solvers to name a few. Recently, there has been much progress in developing and employing the method of lattice Boltzmann equation (LBE) as an alternative, non-traditional computational technique for solving complex fluid dynamic systems. In the LBE approach, one solves the kinetic equation for the particle distribution function and the macroscopic quantities can then be obtained by evaluating the hydrodynamic moments of the distribution function. Other highlighting features of the lattice Boltzmann method (LBM) are:

1. The solution method is local in nature, as the governing equation does not need any information from the neighboring nodes.

2. It employs a stationary, commonly Cartesian, mesh for both fixed and moving boundary problems.

3. The solution process is non-iterative, does not require any matrix inversion, and hence takes minimal computation at each time step.

4. LBE recovers the incompressible Navier-Stokes equations using the Chapman-Enskog expansion.

5. It can be parallelized easily.

In the past, Sui et al. [13] have presented a hybrid immersed-boundary and multi-block LBM which was applied for the simulation of a flapping wing. They studied the two-dimensional hovering of an elliptical shape wing with dragonfly-like kinematics at $R e=157$, and showed good agreement with the results of a previous study. They emphasized that their method not only preserved the advantages of both the immersed-boundary method and LBM, it also improved the accuracy and efficiency by employing a multi-block strategy. Gao et al. $[14,15]$ investigated the ground effect on insect hovering using a two-dimensional immersed boundary-LBM. They examined normal and dragonfly hovering mode at $R e=100$. They showed that, in the normal hovering mode, the symmetry of the flow field is destroyed when the foil moves away from the ground, and the back-stroke is the major contributor to the vertical force. In the dragonfly hovering mode, the ground effect mainly affected the horizontal force when the foil moved away from the ground [14, 15]. Liu, Liu and Lu [16] studied the aerodynamics of the two-dimensional twowinged insect hovering flight and have examined the effect of Reynolds number (25 to 100), wing kinematics (i. e. stroke amplitude and angle of attack), and flight environment (i.e. effect of ground clearance). It was shown that the time-averaged vertical and horizontal forces were weakly dependent on the stroke amplitude as it varied from 3.0 to 5.0, corresponding to the range of amplitude-to-chord ratio employed in the small insect flight [17]. A weak dependency of aerodynamic performance was identified for Reynolds number in the range from to 50 to 100 . Moreover, the aerodynamic forces were significantly enhanced for small ground clearance due to the ground effect. 
More recently, $\mathrm{Wu}$, Shu, and Zhang [18] performed numerical investigation of the flow around two-dimensional hovering elliptical shape with pitching and plunging airfoil at $R e=75$. Their results showed reasonable agreement with the results of previous studies [19, 20]; however, the results simulated delayed rotation showed slight discrepancy when compared with [19, 20]. In addition, LBM has also been applied for simulation of aerodynamics due to forewing and hindwing interaction in gliding dragonfly flight [21]. A multi-block lattice Boltzmann method [22] and second-order accurate treatment for the boundary conditions [23] was employed and a systematic study for angle of attack of forewing $\left(0^{\circ}-6^{\circ}\right)$ and hindwing $\left(6^{\circ}-12^{\circ}\right)$, chord ratios, and different Reynolds numbers $(R e=$ 300,1000 , and 2000) was conducted. The vertical force generated at $R e=300$ was compared with that at $R e=2000$. It was shown that the lift on the wings increased with increasing Reynolds number. Finally, the authors concluded that forewing/hindwing interactions can enhance the total lift force effectively and reduce the drag force on the wings compared to two isolated wings due to triangular camber effect [24].

In spite of these studies, a major challenge in LBM simulations is the handling of no-slip, slip and symmetric boundary conditions. In particular, no-slip boundary treatment on the surface of the wing is at the core of flapping wing simulations. Yu, Mei and Shyy have proposed second-order accurate linear- and quadratic treatments by considering momentum balance in the direction of the discrete velocity set [25]. They have shown smooth variation of force histories compared to other boundary treatments [25]. However, these boundary treatments have not been investigated in the scope of flapping and plunging wing simulations to analyze the accuracy of the solution near the moving boundary. A systematic study is needed that could validate these techniques applied for the simulation of aerodynamics of MAVs and low Reynolds number flapping wing flyers. In addition, the range of $R e=\mathrm{O}\left(10^{2}\right)$ falls in the flight regime of a fruitfly, one of most representative insects investigated in biological flapping research [1, 5 12]. Thus, the objectives of the present study are: 1) assessment of several no-slip boundary treatments of stationary and moving boundaries at $R e=100,2)$ to investigate fluid physics behind zero- and finite-thickness flat plate undergoing pitching and plunging at $R e=O\left(10^{2}\right)$, and 3) to show preliminary results of 3D two-link flexible wing model under pitching and plunging motion.

\section{Methodology and Case Description}

\section{A. Governing equations}

LBM is a simulation technique in which the discretized Boltzmann's equation is solved for the particle distribution function on a regular, uniform Cartesian grid. This discretized form of the equation is derived from the more general Boltzmann's equation that is a function of space, time and phase-space. This Boltzmann's equation is discretized in the phase-vector space that yields the differential form of the LBE that is an equation for the particle distribution function as a function of position and time. The LBE has an inbuilt collision operator that mimics the role of collisions of atoms or molecules in a gas or liquid. The single-time relaxation form of Bhatnagar-GrossKrook (BGK) is the most commonly used form of this collision operator that converts the Boltzmann's equation into a linear differential equation, which could be readily discretized and solved through a time marching procedure. Thus,

$$
\frac{\partial f_{\alpha}}{\partial t}+\mathbf{e}_{\alpha} \cdot \nabla f_{\alpha}=-\frac{\left(f_{\alpha}-f_{\alpha}^{e q}\right)}{\tau}+\Phi_{\alpha}
$$

when discretized in time and discrete-velocity space gives

$$
\frac{f_{\alpha}\left(\mathbf{x}+\mathbf{e}_{\alpha} \delta t, t+\delta t\right)-f_{\alpha}\left(\mathbf{x}+\mathbf{e}_{\alpha} \delta t, t\right)}{\partial t}+\frac{f_{\alpha}\left(\mathbf{x}+\mathbf{e}_{\alpha} \delta t, t\right)-f_{\alpha}(\mathbf{x}, t)}{\partial x}=-\frac{\left(f_{\alpha}(\mathbf{x}, t)-f_{\alpha}^{e q}(\mathbf{x}, t)\right)}{\tau}+\Phi_{\alpha}
$$

Assuming $\delta x=\delta t=1$, Eq. (2) simplifies to the most commonly used 'explicit' lattice Boltzmann equation (LBE), given as

$$
f_{\alpha}\left(\mathbf{x}+\mathbf{e}_{\alpha} \delta t, t+\delta t\right)-f_{\alpha}(\mathbf{x}, t)=-\frac{\left(f_{\alpha}(\mathbf{x}, t)-f_{\alpha}^{e q}(\mathbf{x}, t)\right)}{\tau}+\Phi_{\alpha}
$$


In the LBE, $\tau$ is the dimensionless relaxation time and $\Phi_{\alpha}$ accounts for contributions from any external forces acting on the fluid. $f_{\alpha}^{e q}$ represents the equilibrium distribution function, which depends only on local density and velocity. For a three-dimensional lattice (D3Q19) it can be expressed in the following form:

$$
f_{i}^{e q}=\left\{\begin{array}{cc}
\frac{\rho}{3}\left(1-\frac{\mathbf{u} \cdot \mathbf{u}}{2 c_{s}^{2}}\right) & , i=0 \\
\frac{\rho}{18}\left(1+\frac{\mathbf{e}_{i} \cdot \mathbf{u}}{c_{s}^{2}}+\frac{\left(\mathbf{e}_{i} \cdot \mathbf{u}\right)^{2}}{2 c_{s}^{4}}-\frac{\mathbf{u} \cdot \mathbf{u}}{2 c_{s}^{2}}\right) & , 1 \leq i \leq 6 \\
\frac{\rho}{36}\left(1+\frac{\mathbf{e}_{i} \cdot \mathbf{u}}{c_{s}^{2}}+\frac{\left(\mathbf{e}_{i} \cdot \mathbf{u}\right)^{2}}{2 c_{s}^{4}}-\frac{\mathbf{u} \cdot \mathbf{u}}{2 c_{s}^{2}}\right) & , 7 \leq i \leq 18
\end{array}\right.
$$

where $c_{s}^{2}=1 / 3$ is the speed of sound (in lattice units). Equation (3) is solved as a two-step process. The first step involves the collision step, in which the distributions undergo the following rule:

$$
f_{\alpha}^{\prime}(\mathbf{x}, t)-f_{\alpha}(\mathbf{x}, t)=-\frac{\left(f_{\alpha}(\mathbf{x}, t)-f_{\alpha}^{e q}(\mathbf{x}, t)\right)}{\tau}+\Phi_{\alpha}
$$

The second step involves propagating the distributions to the nearest nodes, and is known as the streaming step:

$$
f_{\alpha}\left(\mathbf{x}+\mathbf{e}_{\alpha} \delta t, t+\delta t\right)=f_{\alpha}^{\prime}(\mathbf{x}, t)
$$

There are many models for the phase-space discretization. Some of the commonly used models are the D2Q9 (twodimensional, 9 speed), D3Q15 (three-dimensional, 15 speed) and D3Q19 (three-dimensional, 19 speed). The zerothand first-order moments of the particle distribution function with the phase space vector at each location yield the macroscopic density and momentum flux, as

$$
p / c_{s}^{2}=\rho=\sum_{\alpha} f_{\alpha} ; \rho \mathbf{u}=\sum_{\alpha} f_{\alpha} \mathbf{e}_{\alpha}
$$

The leading truncation error of a velocity-space discretization is taken into account in a way such that to ensure that the kinematic viscosity in the Navier-Stokes equation derived from Eq. (3) becomes

$$
v=\left(\tau-\frac{1}{2}\right) c_{s}^{2} \delta t
$$

where the positivity of the viscosity requires that $\tau>1 / 2$.

In this work, the D2Q9 and D3Q19 form of the discrete velocity space have been used that yield a square and cubic lattice for the space discretization, respectively. For these models, the phase space velocities are shown in Figure 1. The velocity vectors are given as:

$$
\mathbf{e}_{\alpha}=\left\{\begin{array}{cc}
(0,0), & \alpha=0 \\
( \pm 1,0) c,(0, \pm 1) c, & \alpha=1,2,3,4 \\
( \pm 1, \pm 1) c, & \alpha=5,6,7,8
\end{array}\right.
$$

for the D2Q9 lattice arrangement, and

$$
\mathbf{e}_{\alpha}=\left\{\begin{array}{cc}
(0,0,0), & \alpha=0 ; \\
( \pm 1,0,0) c,(0, \pm 1,0) c,(0,0, \pm 1) c & \alpha=1,2, \cdots, 6 \\
( \pm 1, \pm 1,0) c,( \pm 1,0, \pm 1) c,(0, \pm 1, \pm 1) c & \alpha=7,8, \cdots, 18
\end{array}\right.
$$

for the D3Q19 configuration, where $c$ is the lattice speed and is given by $c=\delta x / \delta t$. 


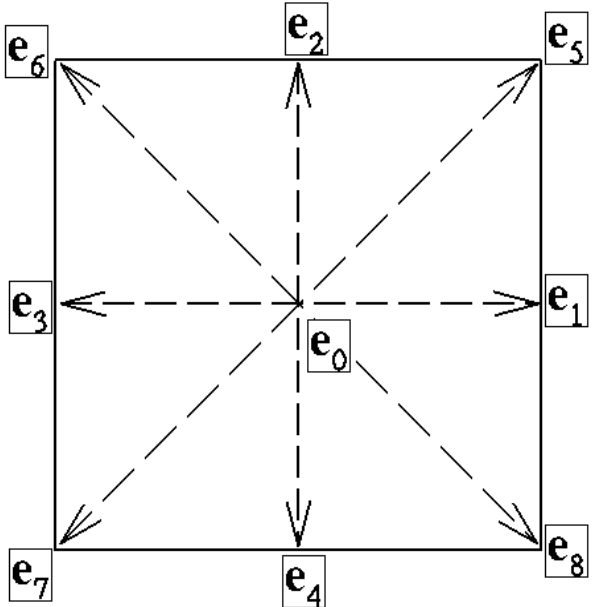

(a) D2Q9

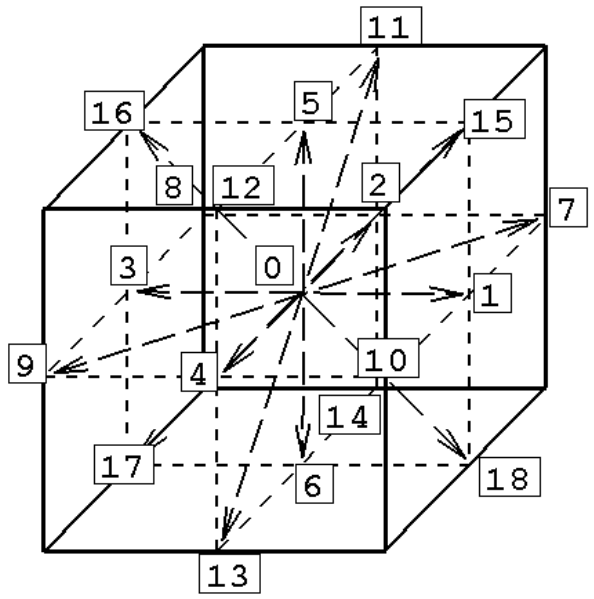

(b) D3Q19

Figure 1: Lattice arrangement for the D2Q9 and D3Q19 discretization.

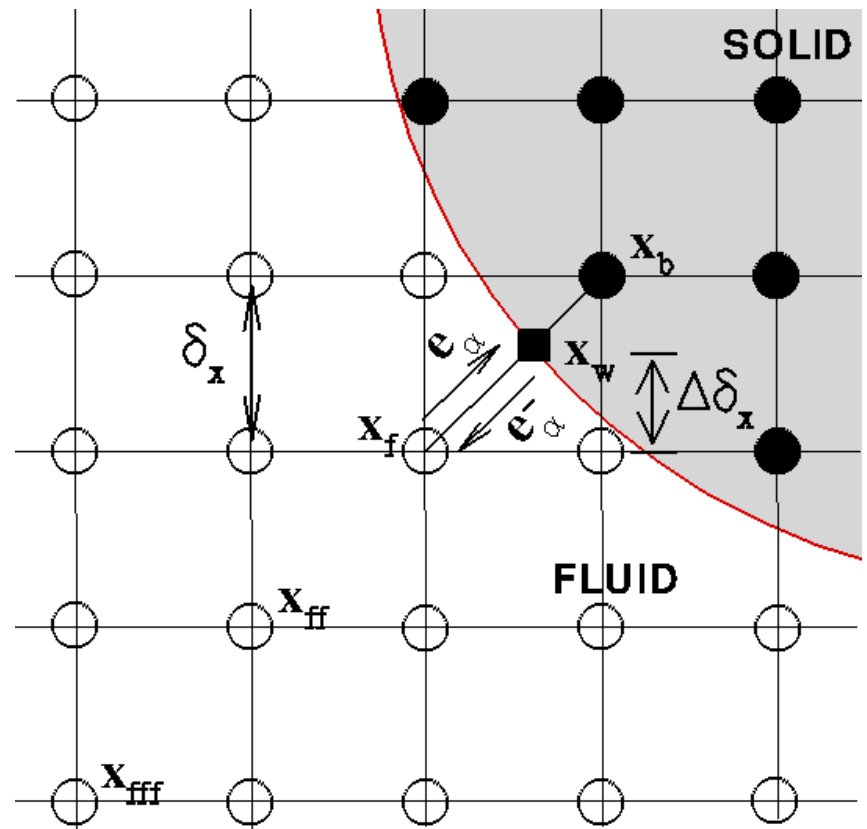

Figure 2: Layout of the regularly spaced lattices and curved wall boundary (solid red). The hollow and filled circles denote fluid and wall nodes respectively. The solid squares denote the boundary nodes inside the solid.

\section{B. Boundary treatments}

Halfway bounceback method

Devising newer and reliable boundary conditions in the BGK variant of the LBE has received considerable attention in the past [22, 23, 25-32]. Some of the boundary conditions commonly encountered in fluid flows are related to stationary or moving wall, inlet or outlet velocity, and inlet/outlet pressure. Since LBM deals with the particle distribution function as the dependent variable, specification of velocity or pressure boundary conditions becomes a highly complex process. In many scenarios, solving for the distribution function based on the macroscopic variables on the boundary could lead to a set of equations that may not have a closed form solution. With regard to such issues, simple rules have been formulated and proposed in literature through which a closure, which is physically harmonious to the problem and at the same time is easy to implement with good accuracy, can be achieved. 
A common method of prescribing the zero-velocity boundary condition at a wall is to reflect the distribution functions opposite to the incoming direction. This technique, however, leads to first-order accuracy at the wall nodes. Ziegler [31] has proposed the shifting of the wall (boundary) half-mesh into the fluid. In other words, the reflection of distribution functions occurs halfway between the nodes, which yields second order accuracy at the wall. This scheme is popularly known as the halfway bounceback method. As is was shown by Ladd [28], and with reference to Figure 2, the distribution functions reflected back from the solid into the fluid nodes can be written as

$$
f_{\bar{\alpha}}\left(\mathbf{x}_{f}\right)=f_{\alpha}\left(\mathbf{x}_{b}\right)+2 w_{\alpha} \rho \frac{3}{c^{2}} \mathbf{e}_{\bar{\alpha}} \cdot \mathbf{u}_{w}
$$

where $w_{\alpha}$ is weight in the direction given by $\alpha, \mathbf{e}_{\bar{\alpha}}$ is the lattice vector opposite to $\mathbf{e}_{\alpha}$ and $\mathbf{u}_{w}$ is the local wall velocity.

The fluid momentum carried by each of the distributions cancels off completely halfway between the wall and fluid nodes, thereby making the boundary treatment second-order accurate. However, the halfway bounceback treatment does not account for any other distance of the wall from the nearest lattice, except $\Delta=1 / 2$. As a result, the true curvilinear object, as shown in Figure 3a, is inherently resolved in a staircase fashion, as shown in Figure $3 b$. This results in loss of accuracy, as a true representation of the object is lost in the process of enforcing the no-slip boundary condition. A remedy for this misrepresentation is to use interpolating boundary conditions that may account for any fractional distance from the fluid nodes.

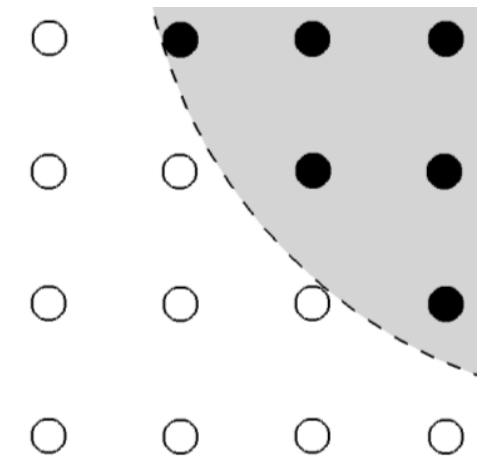

(a) True shape of the solid

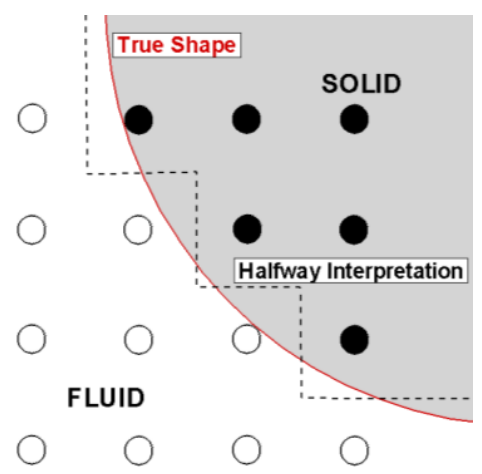

(b) Halfway representation of the solid

Figure 3: The true and halfway bounceback representation of a curved geometry.

\section{Interpolation method}

In this formulation, as shown in figure 2, the location of the wall is recorded and used to calculate the fractional distance of the nearest fluid node from which distribution would be streamed to the corresponding wall node. Hence,

$$
\Delta=\frac{\left|\mathbf{x}_{f}-\mathbf{x}_{w}\right|}{\left|\mathbf{x}_{f}-\mathbf{x}_{b}\right|}
$$

According to the definition, $0 \leq \Delta \leq 1$. In this work, the unified treatment for an irregularly aligned boundary is utilized according to the formulation of $\mathrm{Yu}$ et al. [22]. The linear (first-order expansion) and quadratic (secondorder) interpolation schemes have been used to specify the distribution functions reflected from a moving boundary node into the fluid.

\section{A. First-order}

From Figure 2, after the streaming step the distributions $f_{\alpha}\left(\mathbf{x}_{b}\right)$ and $f_{\alpha}\left(\mathbf{x}_{f}\right)$ are known. Accordingly, the distribution function at the surface of the wall $\left(\mathbf{x}_{w}\right)$ can be approximated using linear interpolation as

$$
f_{\alpha}\left(\mathbf{x}_{w}\right)=f_{\alpha}\left(\mathbf{x}_{f}\right)+\Delta\left[f_{\alpha}\left(\mathbf{x}_{b}\right)-f_{\alpha}\left(\mathbf{x}_{f}\right)\right]
$$

To ensure no-slip condition on the wall, the reflected distribution from the wall node is modified to account for the momentum gained from the moving wall. As a result, 


$$
f_{\bar{\alpha}}\left(\mathbf{x}_{w}\right)=f_{\alpha}\left(\mathbf{x}_{w}\right)+2 w_{\alpha} \rho \frac{3}{c^{2}} \mathbf{e}_{\bar{\alpha}} \cdot \mathbf{u}_{w}
$$

Finally, using $f_{\bar{\alpha}}\left(\mathbf{x}_{w}\right)$ and $f_{\bar{\alpha}}\left(\mathbf{x}_{f}+\mathbf{e}_{\bar{\alpha}}\right)$, the incoming distribution at the fluid node can be written as

$$
f_{\bar{\alpha}}\left(\mathbf{x}_{f}\right)=f_{\bar{\alpha}}\left(\mathbf{x}_{w}\right)+\frac{\Delta}{\Delta+1}\left[f_{\bar{\alpha}}\left(\mathbf{x}_{f}+\mathbf{e}_{\bar{\alpha}}\right)-f_{\bar{\alpha}}\left(\mathbf{x}_{w}\right)\right]
$$

\section{B. Second-order}

The second-order treatment follows a procedure similar to the first-order interpolation. However, the lattice node denoted by $\mathbf{x}_{f f f}=\mathbf{x}_{f}+2 \mathbf{e}_{\bar{\alpha}}$ is also included in the interpolation. Accordingly, the distribution at the wall node is constructed as

$$
f_{\alpha}\left(\mathbf{x}_{w}\right)=f_{\alpha}\left(\mathbf{x}_{f}\right)+\Delta\left[f_{\alpha}\left(\mathbf{x}_{b}\right)-f_{\alpha}\left(\mathbf{x}_{f}\right)\right]+\frac{\Delta(\Delta-1)}{2}\left[f_{\alpha}\left(\mathbf{x}_{b}\right)-2 f_{\alpha}\left(\mathbf{x}_{f}\right)+f_{\alpha}\left(\mathbf{x}_{f}+\mathbf{e}_{\bar{\alpha}}\right)\right]
$$

Using no-slip condition at the moving boundary (i.e. Eq. (13)), the incoming distribution at the fluid node can be written as

$$
f_{\bar{\alpha}}\left(\mathbf{x}_{f}\right)=f_{\bar{\alpha}}\left(\mathbf{x}_{w}\right)+\frac{\Delta}{\Delta+1}\left[f_{\bar{\alpha}}\left(\mathbf{x}_{f}+\mathbf{e}_{\bar{\alpha}}\right)-f_{\bar{\alpha}}\left(\mathbf{x}_{w}\right)\right]-\left[\frac{f_{\bar{\alpha}}\left(\mathbf{x}_{f}+2 \mathbf{e}_{\bar{\alpha}}\right)}{(2+\Delta) / \Delta}-\frac{f_{\bar{\alpha}}\left(\mathbf{x}_{f}+\mathbf{e}_{\bar{\alpha}}\right)}{(1+\Delta) / \Delta}+\frac{\Delta f_{\bar{\alpha}}\left(\mathbf{x}_{w}\right)}{(2+\Delta)(1+\Delta)}\right]
$$

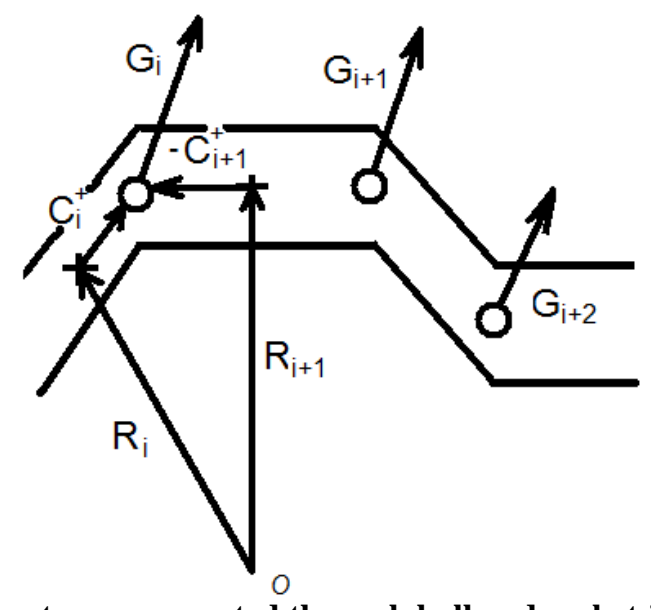

Figure 4: The beam segments are connected through ball and socket joints indicated by circle.

\section{Flexible particle method}

The lattice Boltzmann flexible particle method has been reported by Qi [30] and will be described briefly. In the model, a flexible solid object described as a chain of rigid beam segments are in contact with each other through ball and socket joints [30], as shown in Figure 4. During motion, the following condition has to be satisfied at any time:

$$
\mathbf{R}_{i+1}-\mathbf{C}_{i+1}^{+}=\mathbf{R}_{i}+\mathbf{C}_{i}^{+} \quad i=1, N-1
$$

where $N$ is the total number of beam segments; $i$ is the mass center of the $\mathrm{i}^{\text {th }}$ beam segment; and $\mathbf{R}_{i}$ is the vector from its mass center to the joint $i$, where index $i$ varies from 1 to $N-1$, and the beam segments are labeled by index $i$ in an increasing order. In fact, $\mathbf{C}_{i}^{+}=\mathbf{A}_{i}^{-1} \mathbf{c}_{i}^{+}$, where constant velocity $c_{i}^{+}=\frac{b}{2}\left(\begin{array}{lll}0 & 0 & 1\end{array}\right)$ is the semi-axis of the 
segment, and fixed along the $Z^{\prime}$-direction or the span-wise direction in a body-fixed coordinate system; $\mathbf{A}_{i}$ is the rotational transformation matrix of segment $i$ from the global space coordinate system to the body-fixed coordinate system. The prime sign ' denotes the body-fixed coordinate system. Equation (17) may be rewritten as:

$$
\mathbf{R}_{i+1}-\mathbf{A}_{i+1}^{-1} \mathbf{c}_{i+1}^{+}=\mathbf{R}_{i}+\mathbf{A}_{i}^{-1} \mathbf{c}_{i}^{+} \quad i=1, N-1
$$

The wing, as a whole, can bend and twist through rotation around the ball and socket joints. The bending and twisting moments are proportional to the deformation angles as

$$
\begin{gathered}
\mathbf{T}_{i}^{b}=E I_{\omega} \frac{\theta_{i}-\theta_{i, 0}}{b} \mathbf{n}_{i} \\
\mathbf{T}_{i}^{t}=G I_{p} \frac{\psi_{i}^{\prime}-\psi_{i, 0}^{\prime}}{b} \mathbf{z}_{i} \sigma
\end{gathered}
$$

where $\mathbf{n}_{i}=\left(\mathbf{z}_{i} \times \mathbf{z}_{i+1}\right) /\left|\mathbf{z}_{i} \times \mathbf{z}_{i+1}\right|$ is the unit normal to the bending plane; $\theta_{i, 0}$ is the equilibrium bending angle; $E I_{\omega}$ is the bending flexural rigidity with Young's modulus $E$ and the moment of inertia of the cross-section area $I_{\omega}$, $\sigma=1$ if $\mathbf{x}_{i+1}^{*} \times \mathbf{x}_{i} \cdot \mathbf{z}_{i}>0$, otherwise $\sigma=-1 ; \psi_{i, 0}^{\prime}$ is the equilibrium twisting angle; $G I_{p}$ is torsional rigidity; $G$ is he shear modulus; $I_{p}$ is the torsion constant.

The equations of translational and rotational motion for each constituent segment of the wing may be expressed as:

$$
\begin{gathered}
m_{i} \dot{\mathbf{u}}_{i}=\mathbf{F}_{i}^{h} \\
\mathbf{I}_{m, i} \dot{\mathbf{\Omega}}_{i}=\mathbf{T}_{i}^{h}+\mathbf{T}_{i}^{t}+\mathbf{T}_{i}^{b}
\end{gathered}
$$

where $m_{i}$ is the mass of fiber segment; $\mathbf{F}_{i}^{h}$ is the hydrodynamic force for $\mathrm{i}^{\text {th }}$ segment; $\mathbf{I}_{m, i}$ is the inertial moment matrix; $\mathbf{T}_{i}^{h}$ is hydrodynamic torque; and $\dot{\boldsymbol{\Omega}}_{i}$ is the angular velocity of the fiber segment.

As shown in Figure 4, a constraint force $\left(\mathbf{G}_{i}\right)$ is added at each joint to force the chain of the discretized segment to movie and rotate as a flexible body at each time step. In general, translation and rotation are nonlinear functions of the constraint forces in the constraint Eq. (18). To solve the constraint forces, displacement and rotation of fiber segments are expanded, upto second-order, in a power series of time step. In the half leap-frog algorithm [32], for translation, the position of the mass center of the segment $i$ can be written as

$$
\mathbf{R}_{i}(t+\Delta t)=\mathbf{R}_{i}(t)+\Delta t\left[\dot{\mathbf{R}}_{i}\left(t-\frac{\Delta t}{2}\right)+\frac{\Delta t}{m} \mathbf{F}_{i}^{h}(t)\right]+\frac{(\Delta t)^{2}}{m}\left(\mathbf{G}_{i}-\mathbf{G}_{i-1}\right)
$$

where $\dot{\mathbf{R}}_{i}$ is the velocity of mass center of segment at $t-\Delta t / 2$ and $\Delta t$ is the time step. It should be noted that the same formulas could be written for other segments with the corresponding index. For rotation of the segment, the rotational transformational matrix $\left(\mathbf{A}_{i}\right)$ from the space coordinate to the body-fixed coordinate is associated with quaternion $\mathbf{Q}_{i}=\left\{q_{i 0}, q_{i x}, q_{i y}, q_{i z}\right\}^{T}$ by

$$
\mathbf{A}_{i}=\left(\begin{array}{ccc}
q_{i 0}^{2}+q_{i x}^{2}-q_{i y}^{2}-q_{i z}^{2} & 2\left(q_{i x} q_{i y}+q_{i 0} q_{i z}\right) & 2\left(q_{i x} q_{i z}-q_{i 0} q_{i y}\right) \\
2\left(q_{i x} q_{i y}-q_{i 0} q_{i z}\right) & q_{i 0}^{2}-q_{i x}^{2}+q_{i y}^{2}-q_{i z}^{2} & 2\left(q_{i y} q_{i z}+q_{i 0} q_{i x}\right) \\
2\left(q_{i x} q_{i z}+q_{i 0} q_{i y}\right) & 2\left(q_{i y} q_{i z}-q_{i 0} q_{i x}\right) & q_{i 0}^{2}-q_{i x}^{2}-q_{i y}^{2}+q_{i z}^{2}
\end{array}\right)
$$

Euler angles of each segment $\left(\phi_{i}, \psi_{i}\right.$, and $\left.\theta_{i}\right)$ can be directly related to the quaternion [33] by 


$$
\begin{aligned}
& q_{i 0}=\cos \left(\frac{1}{2}\left(\phi_{i}+\psi_{i}\right)\right) \cos \left(\frac{1}{2} \theta_{i}\right) \\
& q_{i x}=\cos \left(\frac{1}{2}\left(\phi_{i}-\psi_{i}\right)\right) \sin \left(\frac{1}{2} \theta_{i}\right) \\
& q_{i y}=\sin \left(\frac{1}{2}\left(\phi_{i}-\psi_{i}\right)\right) \sin \left(\frac{1}{2} \theta_{i}\right) \\
& q_{i z}=\sin \left(\frac{1}{2}\left(\phi_{i}+\psi_{i}\right)\right) \cos \left(\frac{1}{2} \theta_{i}\right)
\end{aligned}
$$

A leap-frog algorithm [33] can be applied to the current problem with constraint forces. First, the angular moment $\mathbf{J}_{i}$ is introduced from time $t-\Delta t / 2$ to time $t$,

$$
\mathbf{J}_{i}(t)=\mathbf{J}_{i}^{\prime}(t)+\frac{\Delta t}{2} \mathbf{T}_{i}^{c}(t)
$$

where $\mathbf{J}_{i}^{\prime}(t)=\mathbf{J}_{i}(t-\Delta t / 2)+\frac{\Delta t}{2} \mathbf{T}_{i}(t)$ is the angular moment without the constraint force at time $t$, while the constraint torque for segment $i$ is $\mathbf{T}_{i}^{c}(t)=\mathbf{C}_{i}^{+} \times \mathbf{G}_{i}^{+}+\mathbf{C}_{i}^{+} \times \mathbf{G}_{i-1}^{+}$. The angular velocity $\boldsymbol{\omega}_{b i}$ in the body- fixed coordinate could be obtained by

$$
\boldsymbol{\omega}_{b i}(t)=\mathbf{I}^{-1} \mathbf{A}_{i}(t) \mathbf{J}_{i}(t)
$$

This angular velocity $\left(\boldsymbol{\omega}_{b i}\right)$ could be computed through the following equation

where

$$
\dot{\mathbf{Q}}_{i}(t)=\mathbf{B}\left(\mathbf{Q}_{i}(t)\right)\left(\begin{array}{c}
0 \\
\boldsymbol{\omega}_{b i}(t)
\end{array}\right)
$$

$$
\mathbf{B}\left(\mathbf{Q}_{i}(t)\right)=\frac{1}{2}\left(\begin{array}{cccc}
q_{i 0} & -q_{i x} & -q_{i y} & -q_{i z} \\
q_{i x} & q_{i 0} & -q_{i z} & q_{i y} \\
q_{i y} & q_{i z} & q_{i 0} & -q_{i x} \\
q_{i z} & -q_{i y} & q_{i x} & q_{i 0}
\end{array}\right)
$$

Furthermore, it is known that

$$
\mathbf{Q}_{i}\left(t+\frac{\Delta t}{2}\right)=\mathbf{Q}_{i}(t)+\frac{\Delta t}{2} \dot{\mathbf{Q}}_{i}(t)
$$

Substituting Eq. (26) into Eq. (28), and using Eqs. (24) and (25), it can be rewritten as:

$$
\mathbf{Q}_{i}\left(t+\frac{\Delta t}{2}\right)=\mathbf{Q}_{i}(t)+\frac{\Delta t}{2} \mathbf{C}_{i}(t)+\frac{\Delta t^{2}}{4} \mathbf{E}_{i}(t)
$$

Where

$$
\mathbf{C}_{i}(t)=\mathbf{B}\left(\mathbf{Q}_{i}(t)\right)\left(\begin{array}{c}
0 \\
\mathbf{I}^{-1} \mathbf{A}_{i}(t) \dot{\mathbf{J}}_{i}(t)
\end{array}\right)
$$

10

American Institute of Aeronautics and Astronautics 
and

$$
\mathbf{E}_{i}(t)=\mathbf{B}\left(\mathbf{Q}_{i}(t)\right)\left(\begin{array}{c}
0 \\
\mathbf{I}^{-1} \mathbf{A}_{i}(t) \mathbf{T}_{i}^{c}(t)
\end{array}\right)
$$

The angular moment at a half time step $\left(t+\frac{\Delta t}{2}\right)$ is

$$
\mathbf{J}_{i}\left(t+\frac{\Delta t}{2}\right)=\mathbf{J}_{i}^{\prime}\left(t+\frac{\Delta t}{2}\right)+\Delta t \mathbf{T}_{i}^{c}(t)
$$

where $\mathbf{J}_{i}^{\prime}\left(t+\frac{\Delta t}{2}\right)=\mathbf{J}_{i}\left(t-\frac{\Delta t}{2}\right)+\Delta t \mathbf{T}_{i}(t)$. The angular velocity $\boldsymbol{\omega}_{b i}$ at a half time step in the body-fixed coordinate is

$$
\boldsymbol{\omega}_{b i}\left(t+\frac{\Delta t}{2}\right)=\mathbf{I}^{-1} \mathbf{A}_{i}\left(t+\frac{\Delta t}{2}\right) \mathbf{J}_{i}\left(t+\frac{\Delta t}{2}\right)
$$

Using Eq. (26) again at $t+\frac{\Delta t}{2}$ we compute

$$
\dot{\mathbf{Q}}_{i}\left(t+\frac{\Delta t}{2}\right)=\left(\mathbf{B}\left(\mathbf{Q}_{i}(t)\right)+\frac{\Delta t}{2} \mathbf{B}_{\mathbf{i}}\left(\mathbf{C}_{i}(t)\right)+\frac{(\Delta t)^{2}}{4} \mathbf{B}_{\mathbf{i}}\left(\mathbf{E}_{i}(t)\right)\right)\left(\begin{array}{c}
0 \\
\boldsymbol{\omega}_{b i}\left(t+\frac{\Delta t}{2}\right)
\end{array}\right)
$$

while $\mathbf{A}_{i}\left(t+\frac{\Delta t}{2}\right)$ in Eq. (33) could be calculated by applying Eq. (22) at $t+\frac{\Delta t}{2}$. It is noted that the term $\mathbf{E}_{i}(t)$ in the right hand of Eq. (34) can be ignored as it leads to higher order terms in the later equations. Moreover,

$$
\mathbf{Q}_{i}(t+\Delta t)=\mathbf{Q}_{i}(t)+\Delta t \dot{\mathbf{Q}}_{i}\left(t+\frac{\Delta t}{2}\right)
$$

Using Eq. (33) and substituting Eq. (34) into Eq. (35), it may be rewritten as:

$$
\mathbf{Q}_{i}(t+h)=\mathbf{Q}_{i}(t)+\Delta t \mathbf{E}_{1 i}+\frac{\Delta t}{2} \mathbf{E}_{2 i}+\Delta t^{2} \mathbf{E}_{3 i}
$$

where

$$
\begin{gathered}
\mathbf{E}_{1 i}=\mathbf{B}\left(\mathbf{Q}_{i}(t)\right)\left(\begin{array}{c}
0 \\
\mathbf{I}^{-1} \mathbf{A}_{i}\left(t+\frac{\Delta t}{2}\right) \dot{\mathbf{J}}_{l}\left(t+\frac{\Delta t}{2}\right)
\end{array}\right) \\
\mathbf{E}_{2 i}=\mathbf{B}\left(\mathbf{C}_{i}(t)\right)\left(\begin{array}{c}
0 \\
\mathbf{I}^{-1} \mathbf{A}_{i}\left(t+\frac{\Delta t}{2}\right) \dot{\boldsymbol{J}}_{l}\left(t+\frac{\Delta t}{2}\right)
\end{array}\right) \\
\mathbf{E}_{3 i}=\boldsymbol{B}\left(\boldsymbol{Q}_{i}(t)\right)\left(\begin{array}{c}
0 \\
\mathbf{I}^{-1} \mathbf{A}_{i}\left(t+\frac{\Delta t}{2}\right) \mathbf{T}_{i}^{c}(t)
\end{array}\right)
\end{gathered}
$$

During the procedure described earlier, the accuracy is kept up to the second order in $\Delta t$, and any higher order terms than two are truncated. The quaternion should be normalized before substituting into Eq. (22) so that $\mathbf{Q}_{i} \cdot$ $\mathbf{Q}_{i}=1$ is satisfied. $\mathbf{Q}_{i}(t+\Delta t)$ can then be substituted into Eq. (18) to resolve the constraint force $\mathbf{G}_{i}$. 


\section{Case descriptions}

\section{Zero thickness flat plate}

a. Stationary zero thickness flat plate with zero angle of attack at $R e=100$

In this study, flow past a stationary plate at $R e=U_{\infty} c / v=100$ has been simulated using different ways of handling the no-slip boundary condition on the wall. Figure 6 shows the schematic with the domain and boundary conditions enforced on each boundary of the two-dimensional setup. The domain was discretized into $N_{x} \times N_{y}=$ $1600 \times 800$ lattices. The length of the plate was equivalent to 40 lattice nodes. At the inlet, uniform flow was prescribed based on the method of bounceback of the non-equilibrium distribution function [34]. At the outlet, an extrapolation scheme was used to determine the incoming distribution functions:

$$
f_{\alpha}\left(N_{x}, j\right)=2 f_{\alpha}\left(N_{x}-1, j\right)-f_{\alpha}\left(N_{x}-2, j\right) \quad \forall \alpha=3,6,7
$$

Symmetry boundary conditions were applied at the top and bottom faces of the domain. The role of the location of the wall and the its handling by the different LBM treatments was also analyzed by placing the wall at different distances from the nearest lattice. An in-house Navier-Stokes based solver [6] has been used for the purpose of validating the lattice Boltzmann results.

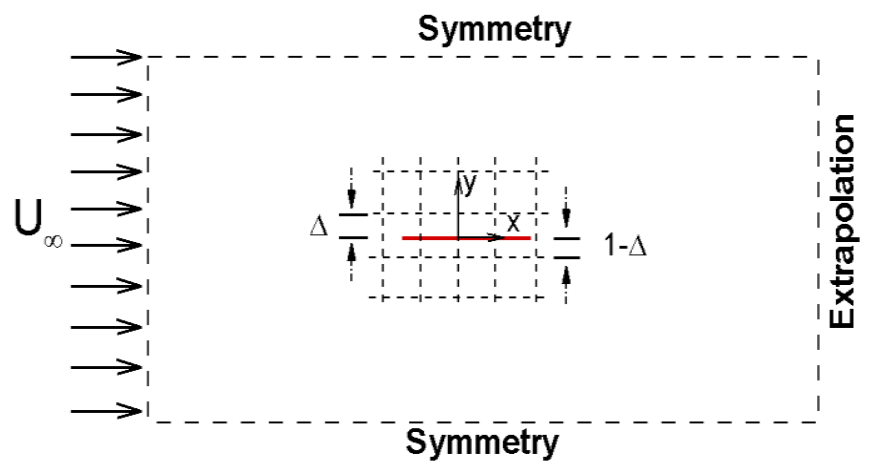

Figure 5: Flow past a stationary plate (shown in red) with the enforced boundary conditions.

b. Vertically oscillating plate with zero angle of attack at $R e=100$

In addition to the earlier problem, flow past a vertically oscillating plate at $\operatorname{Re}\left(=U_{\infty} L / v\right)=100$ has also been simulated for different amplitudes of the plate, keeping the frequency at a fixed value. This allows to analyze the role of temporally varying distance of the plate from the nearest lattices for different schemes of the no-slip boundary condition on the solid wall. The schematic with the domain and the direction of motion of the plate is shown in Figure 6. The domain was discretized into $N_{x} \times N_{y}=4000 \times 800$ lattices, and similar boundary conditions as earlier were enforced on each of the outer boundaries. The plate, which comprised of 40 lattice nodes, was placed at $y=N_{y} / 2$ at time $t=0$. The displacement motion of the oscillating plate (from the initial position) was prescribed based on the following kinematics:

$$
y(t)=y_{a} \sin (2 \pi f t)
$$

where $y_{a}$ and $f$ are the amplitude and frequency, respectively, that define the motion of the plate.

Two different of amplitudes were considered in this study: $y_{a}=0.49 \delta_{x}$ and $y_{a}=0.51 \delta_{x}$. The former ensures that the plate remains confined within a particular lattice at all times, whereas the latter makes sure that the plate crosses the lattice when $y(t)=0.5 \delta_{x}$. 


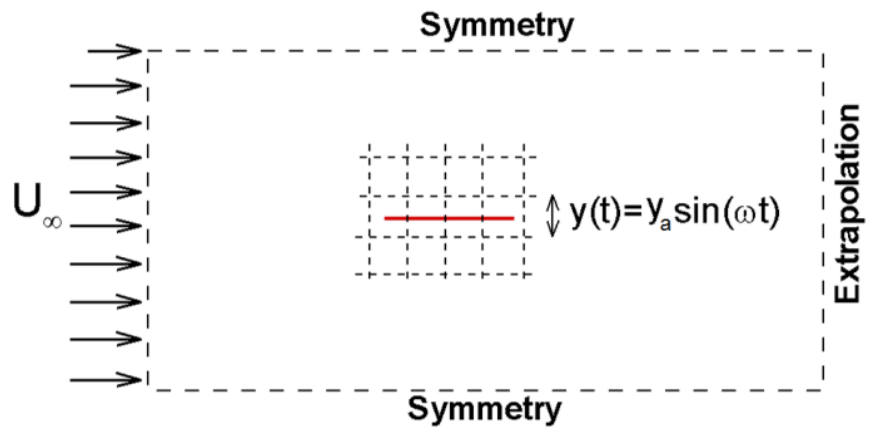

Figure 6: Flow past a vertically oscillating plate (shown in red) with the kinematics and boundary conditions.

c. Three-dimensional wing hovering with delayed rotation at $\mathrm{Re}=100$

The simplified kinematics governing the rotational (pitching) and translational (plunging) motion of a rigid zerothickness wing adopted in this work are described in Eq. (39). The rotational motion is governed by the flapping frequency $(f)$ and the angular amplitude $\left(\alpha_{a}\right)$, whereas the translational motion depends on the plunging amplitude $\left(x_{a}\right)$ and the flapping frequency.

$$
\begin{gathered}
\alpha(t)=\alpha_{0}-\alpha_{a} \sin (2 \pi f t+\phi) \\
x(t)=x_{a} \sin (2 \pi f t)
\end{gathered}
$$

For the delayed rotation case simulated in this work, the following parameters were chosen for direct comparison of results obtained through a finite-volume Navier-Stokes solver [34]: $2 x_{a} / c=2.0, \alpha_{0}=90^{\circ}, \alpha_{a}=45^{\circ}, \phi=60^{\circ}$. The Reynolds number is defined based on the maximum translational velocity and the chord width, and is given as $R e=\frac{U_{\max } c}{v}$. The wing position and orientation for this set of governing parameters is shown in Figure 7 for both forward and backward strokes. The simulation domain comprised of $251 \times 251 \times 251$ lattice nodes, and the aspect ratio of the wing was fixed at $\frac{b^{2}}{b c}=4$, based on the span and the chord lengths. The chord length was equivalent to 40 lattice nodes.

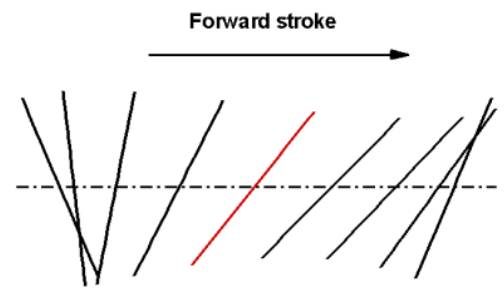

(a) Forward stroke

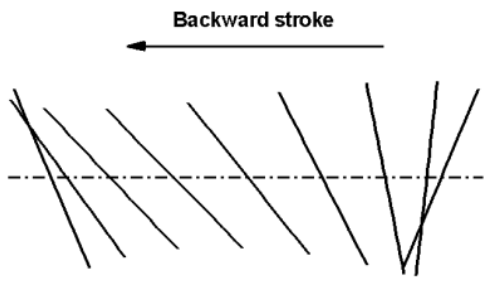

(b) Backward stroke

Figure 7: Forward and backward strokes, with the orientation of the wing, as described by Eq. (39).

\section{Finite thickness flat plate}

a. Three-dimensional wing hovering with advanced rotation at $R e=136$

For three-dimensional finite thickness flat plate with the advanced rotation case, the following parameters presented in Eq. (39) were chosen for direct comparison with the Navier-Stokes solutions [34]: $2 x_{a} / c=0.6, \alpha_{0}=$ $90^{\circ}, \alpha_{a}=45^{\circ}, \phi=45^{\circ}$, and $f=0.8 \mathrm{~Hz}$. The Reynolds number was 136 and the aspect ratio of the wing was 2.5 . 
The thickness of the plate is $7.5 \%$ of chord. A cylinder simulation box was used and the radius of the cylinder is 500 lattice nodes. The chord length was equivalent to 40 lattice nodes. The flow velocity in $Z$ direction (i.e. spanwise direction) and velocity gradient in $X$ and $Y$ directions on symmetrical plane were set to zero. No-slip boundary condition was enforced using the halfway bounceback method.

b. Three-dimensional flexible wing hovering with advanced rotation at $R e=100$

For the three-dimensional flexible pitching and plunging wing case simulated in this work, the prescribed motion of the plate described by Tommey and Eldredge [35] was followed. The kinematics could be mathematically described as

$$
\begin{gathered}
\alpha(t)=-\alpha_{a} \frac{G_{r}(f t)}{\max G_{r}} \\
x(t)=x_{a} \frac{G_{t}(f t)}{\max G_{r}} C(f t)
\end{gathered}
$$

where the rotational shape function is $G_{r}(t)=\tanh \left[\sigma_{r} \cos (2 \pi t+\phi)\right]$ and the translational shape function is $G_{t}(t)=\int_{t} \tanh \left[\sigma_{t} \cos \left(2 \pi t^{\prime}\right)\right] d t^{\prime}$, with the start-up conditioner given by $C(t)=\frac{\tanh (8 t-2)+\tanh 2}{1+\tanh 2}$. In this study, the following parameters were chosen for a direct comparison with the published data $[35]: x_{a} / c=2.8,, \alpha_{a}=$ $45^{\circ}, \phi=45^{\circ}, \sigma_{r}=1.885$, and $\sigma_{t}=1.885$. The Reynolds number, based on the peak angular velocity, was 100 . Two simulation domains were considered: $10 \times 382 \times 384$ and $15 \times 576 \times 576$ lattice nodes. An equivalent chord length of 48 and 72 lattice nodes was used for each simulation. The two-dimensional simulations [35] used a wing with an infinitely long span. Although the present simulations were conducted in three-dimensions, an infinite span was imitated by choosing a span that covered the entire length in the periodic direction. Solid walls were set at the bottom and top boundaries of the simulation box in the vertical or $Y$-direction. Periodicity was applied in the horizontal and span directions. The total number of the segments in the flexible particle model was set at $N=2$ and the same damping force as that in Tommey and Eldredge [35] was incorporated. The two-segment wing had the same set-up as Tommey and Eldredge [35] and underwent flapping at the center of the simulation box.

\section{Results and Discussions}

\section{Zero thickness flat plate in free stream at $R e=100$}

We first visit the two-dimensional problem of uniform flow past a stationary zero thickness flat plate at $R e=100$. The motivation behind selecting the zero-thickness plate for LBM simulations can be explained as follows. Commonly, LBM employs the use of a Cartesian, stationary, and non-adaptive grid on which the evolution of the distribution function occurs. For flows related to movement of a solid object with some thickness (for example, sedimentation of a sphere and hovering of a finite-thickness plate), a lattice, that was confined to the interior of the solid object at an earlier time step, can, all of a sudden, be exposed to the fluid side of the domain. In such a case, fluid mass needs to be 'created' at this newly emerged node, and mass 'destroyed' at the node that may have entered the solid object $[23,28]$. A method commonly adopted to create mass on this newly fluid node involves prescribing distribution that equals the equilibrium function corresponding to the solid velocity and mean fluid density [28]. However, this may lead to large fluctuations in the pressure field, which manifest in the form of sudden jumps in the aerodynamic forces [23]. Thus, to avoid this frequent creation and destruction of mass during the course of the simulation, the results in this section rely on zero-thickness solid objects. The velocity profiles near the surface of the plate computed using LBM have been compared with those obtained through a finite-volume based NavierStokes solver [6].

\section{A. Stationary and zero angle of attack}

Flow past a stationary flat plate aligned with the direction of incoming flow is simulated at $R e=100$. The description of the domain and the boundary conditions was provided in sub-section D of section II. Typically in LBM, aerodynamic forces due to the interaction of a stationary or moving wall with a fluid can be computed based 
on the momentum exchange [25,27,28] or the stress-integration [27] methods. As the surface of a solid may not necessarily be aligned with the fluid nodes at any given time instant, the stress integration method involves extrapolation of pressure and shear stress from the latter to the former. This renders the computation difficult to implement, especially for a rapidly moving boundary. Due to its local operation and ease of implementation, the current study employs the momentum exchange method for the calculation of forces. In Table 1, a comparison of the steady state drag coefficient computed using Navier-Stokes (NS) and different LBM wall treatments is shown. Drag coefficient, computed in LBM using bounceback, first-and second-order interpolation, showed a deviation from the NS solution by less than $5 \%$. The stream-wise velocity profile as a function of the distance normal to the flat plate, at its mid-point, is shown in Figure 8 (a). From Figure 8(a), it can be observed that velocity profile in the region close to the solid plate, when the plate is placed in the middle of the lattices (i.e. $\Delta=1 / 2$ ), computed using the bounce back, first-order and second-order interpolations is in excellent agreement with the NS solution. Similar simulations were also conducted with the plate placed at locations other than halfway between the lattice, and the $\mathrm{L}_{2}$ error norm for the velocity obtained using halfway bounceback and second-order interpolation for two other positions $\left(\Delta=1 / 4\right.$ and 3/8) are shown in Figure $8(b)$. The $\mathrm{L}_{2}$ norm was defined as

$$
L_{2}=\frac{\left[\sum_{y}\left(u_{L B M}(y)-u_{N S}(y)\right)^{2}\right]^{1 / 2}}{\left[\sum_{y}\left(u_{N S}(y)^{2}\right)\right]^{1 / 2}}
$$

The profile obtained through second-order interpolation with the wall placed at different locations indicates good agreement with the NS solution. Although the velocity profile obtained through halfway bounceback matches well with second-order interpolation for $\Delta=1 / 2$, the former shows significant deviation when compared to cases where the wall location is not halfway between the lattice as is shown in Figure 8 (b). As a result, accuracy of the flow field can be compromised when using halfway bounceback to enforce the no-slip boundary condition near the plate for any general $\Delta \neq 1 / 2$.

Table 1: Comparison of drag coefficient between LBM and NS solutions at $R e=100$

\begin{tabular}{ll}
\hline \hline & $C_{D}$ \\
\hline \hline Navier-Stokes & 0.343 \\
Halfway bounceback & 0.362 \\
Linear Interpolation & 0.360 \\
Second-order Interpolation & 0.358 \\
\hline \hline
\end{tabular}
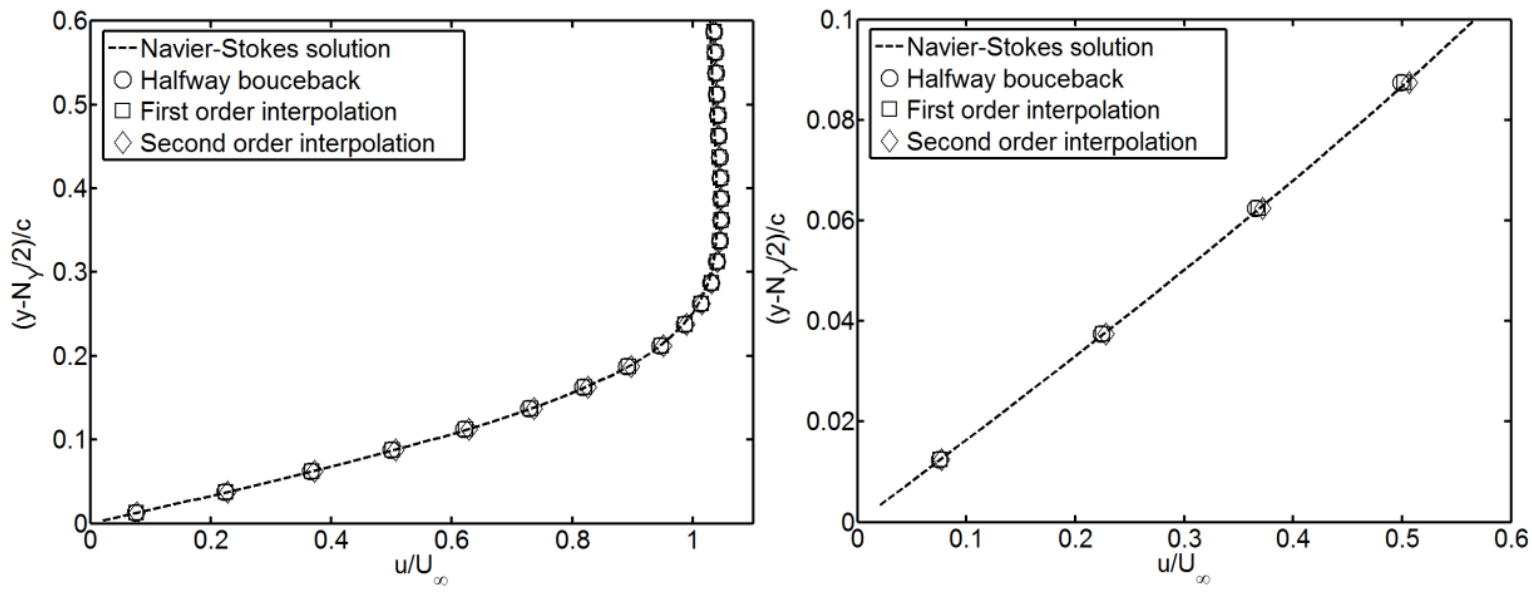

(a) Horizontal velocity profile as a function of distance away from the stationary plate with Navier-Stokes and different lattice Boltzmann treatments for $\Delta=1 / 2$. 


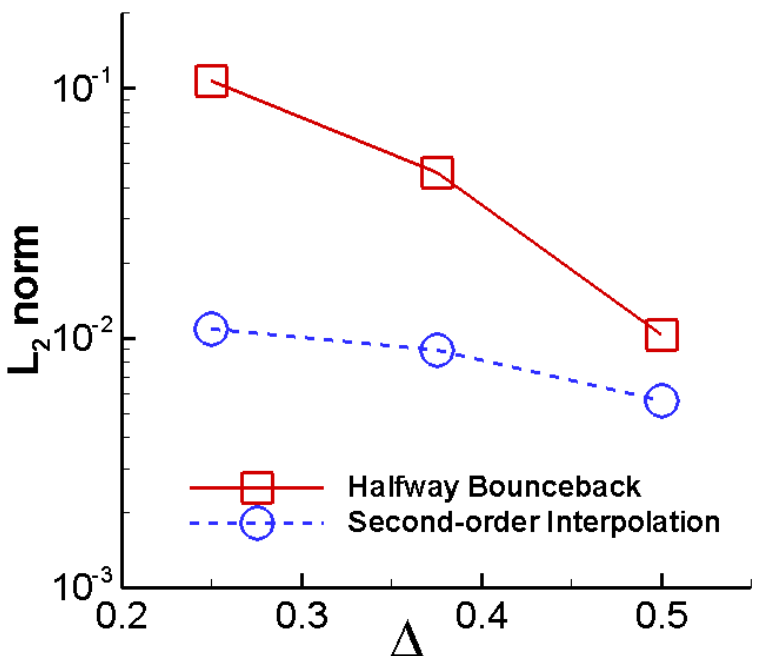

(b) $\mathbf{L}_{2}$ norm calculated for horizontal velocity as a function of fractional distance away from fluid node with halfway bounceback and second-order interpolation.

Figure 8: Comparison of stream-wise velocity profile near the flat plate at the mid-point for $R e=100$ obtained through Navier-Stokes, halfway bounceback, first and second-order interpolations methods.

\section{B. Vertically oscillating and zero angle of attack}

We now address the two-dimensional problem of uniform flow past an oscillating, zero thickness flat plate at $R e=100$. Details regarding the simulation setup were given in sub-section D of section II. The aerodynamic forces computed using different boundary treatments in the context of LBM have been compared with each other.

\section{B.1 Amplitude of oscillating plate: $y_{a}=0.49 \delta_{x}$}

We first consider the case of a vertically oscillating plate with an amplitude given by $y_{a}=0.49 \delta_{x}$, and at a frequency given by $f \delta t=10^{-4}$. This amplitude ensures that the plate remains confined within the cells given by $j=N_{y} / 2-1$ and $j=N_{y} / 2+1$. As a result, the momentum transfer occurs only along links in a certain direction. For example, at node ' $A$ ' in Figure 9(a), links 4, 7 and 8 will undergo bounce-back, and at 'B' nodes 2, 5 and 6 will be bounced-back. This occurs for the course of the simulation. A comparison of the instantaneous drag and lift coefficients for different lattice Boltzmann boundary treatments is shown in Figure 10. The dotted line in the figure indicates the displacement of the plate as a function of time, given by Eq. (38). It was found that for all solid-fluid boundary treatments considered, the drag and lift forces showed no fluctuation (or noise) when recorded over many cycles.

\section{B.2 Amplitude of oscillating plate: $y_{a}=0.51 \delta_{x}$}

The drag and lift coefficient for a slightly higher amplitude of oscillation $\left(y_{a}\right)$ of the flat plate are shown in Figure 11. Again, the dotted line in the figure indicates the displacement of the plate as a function of time, given by Eq. (38). Drag and lift forces show high fluctuations and non-smoothness at the exact time instant when the plate crosses into the adjoining cell, indicated by the time instants corresponding to $y(t)= \pm 0.5 \delta_{x}$. For instance, in Figure 11(a), the drag coefficient shows an increase from $C_{D}=0.36$ to $C_{D}=2.35$ for halfway bounceback method at this time instant. This crossing of the nodes results in reversal of the direction of momentum transfer from the moving boundary. Unlike the previous case, the momentum transfer occurs along link directions that are dynamic and change with the position of the plate. As shown in Figure 9, for instance, at node 'A' links 4, 7 and 8 will undergo bounce-back at time $t=t_{1}$. If the plate crosses into the adjoining cell at $t=t_{1}+\delta t$, the link directions will be reversed, and links 2, 5 and 6 will be involved in momentum exchange. As shown in Figure 11, the use of interpolation results in lowering of the magnitude of fluctuation i.e. from $C_{D}=2.35$ (for halfway bounceback) to $C_{D}=0.73$ and $C_{D}=0.72$ using linear and quadratic interpolation, respectively. However, interpolation could not eliminate these fluctuations. Figure 12 shows pressure and vorticity contours around the plate before and after the 
plate has crossed the lattice to investigate its effect on pressure and velocity around a moving boundary. It can be seen that vorticity contour at two time instants are very similar while pressure distributions near the plate show the presence of a large low pressure region when the plate has crossed the lattice. The appearance of this low pressure region explains the sudden increase or decrease in force coefficients at this very time instant. Earlier studies have speculated that the source of fluctuations in LBM could be attributed to the approximation of the distribution function on the new fluid nodes through an equilibrium distribution [22, 31]. Since the current simulations deal with solid objects of zero-thickness, it has been guaranteed that the creation of new distribution functions is not the source of fluctuations so commonly observed in LBM. Results of the present study indicate that the crossing of the plate and reversal of the bounce-back links is the main source of large fluctuations in the forces.

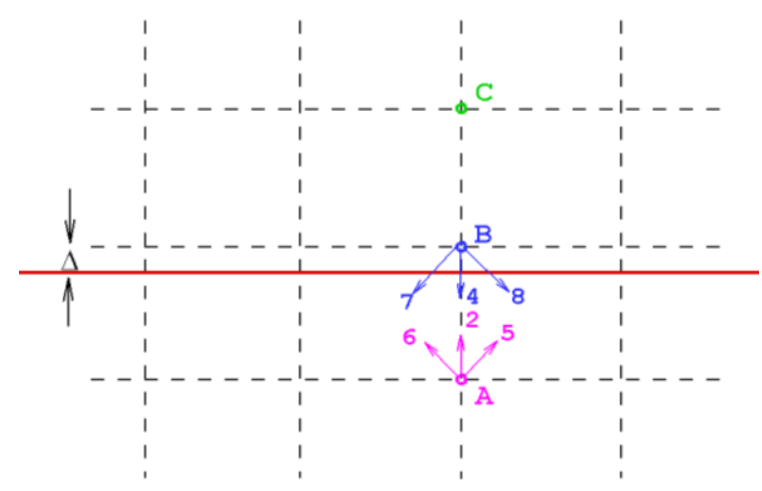

(a) $t=t_{1}$

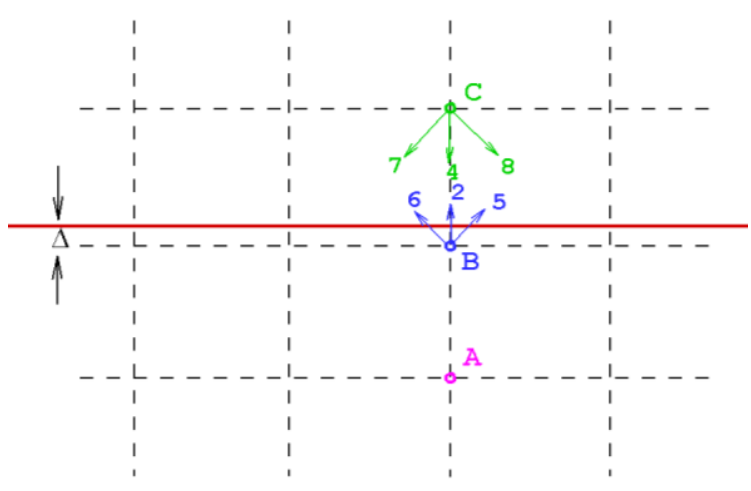

(a) $t=t_{1}+\delta t$

Figure 9: Link bounceback at (a) time $t=t_{1}$ before the plate (shown in red) has crossed into the cell B-C, and (b) time $t=t_{1}+\delta t$ just after the plate has crossed into the cell B-C.

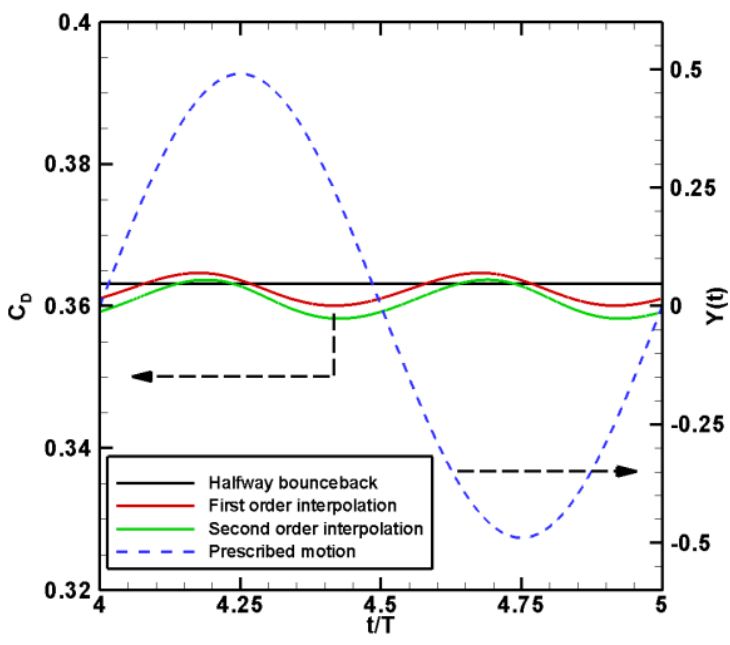

(a) Drag coefficient

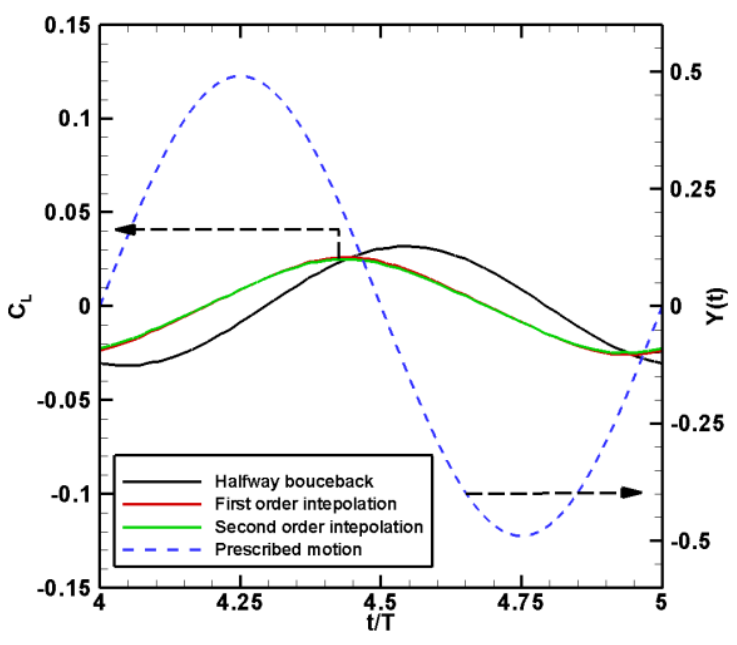

(b) Lift coefficient

Figure 10: Comparison of instantaneous drag and lift coefficient of LBM solutions between different boundary treatments $\left(R e=100\right.$ and $\left.y_{a}=0.49 \delta_{x}\right)$. The dotted blue line indicates the kinematics of the oscillating plate. 


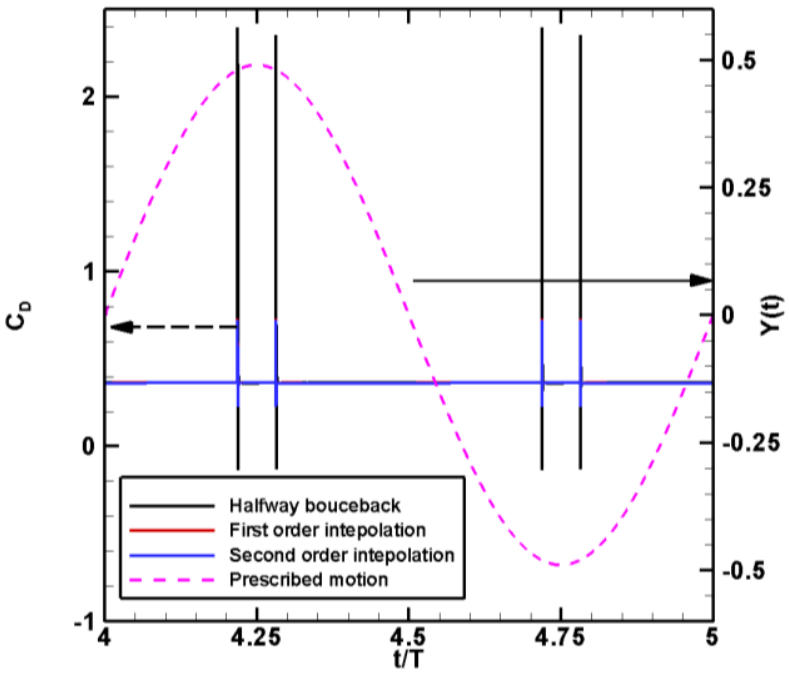

(a) Drag coefficient

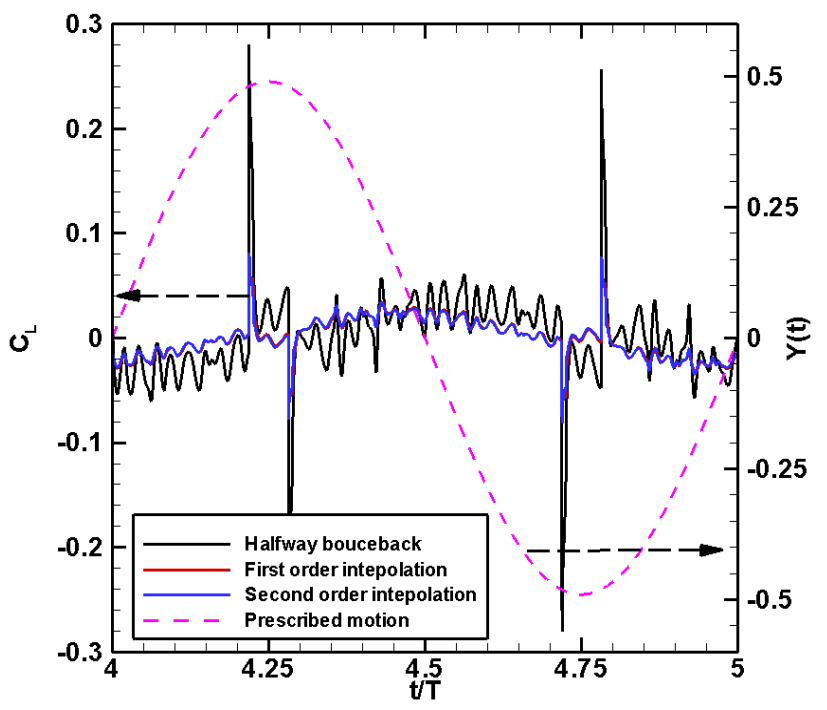

(b) Lift coefficient

Figure 11: Comparison of instantaneous drag and lift coefficient of LBM solutions between different boundary treatments $\left(\operatorname{Re}=100\right.$ and $\left.y_{a}=\mathbf{0 . 5 1} \delta_{x}\right)$. The dotted blue line indicates the kinematics of the oscillating plate.

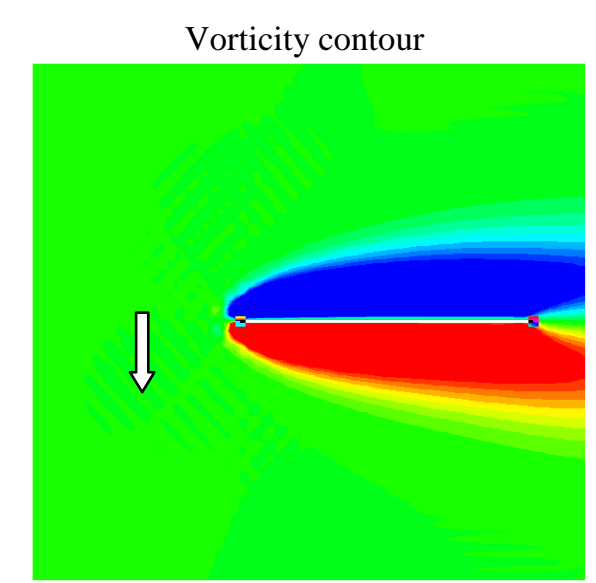

Pressure contour

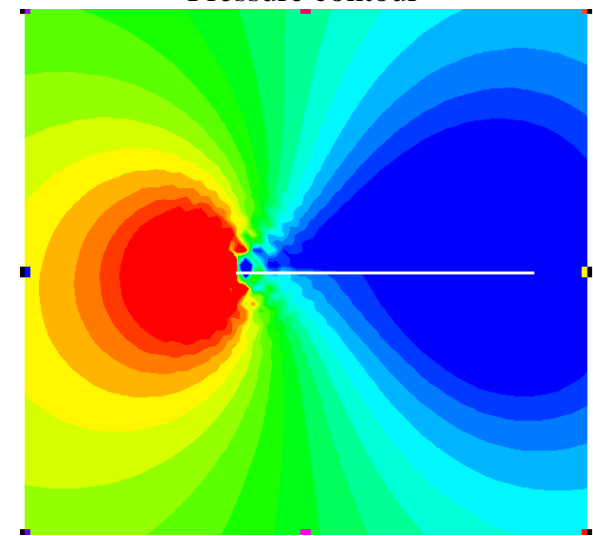

(1) Before crossing

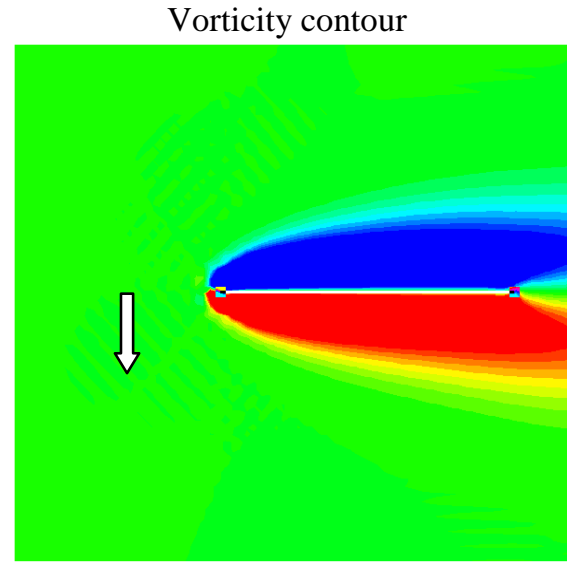

Pressure contour

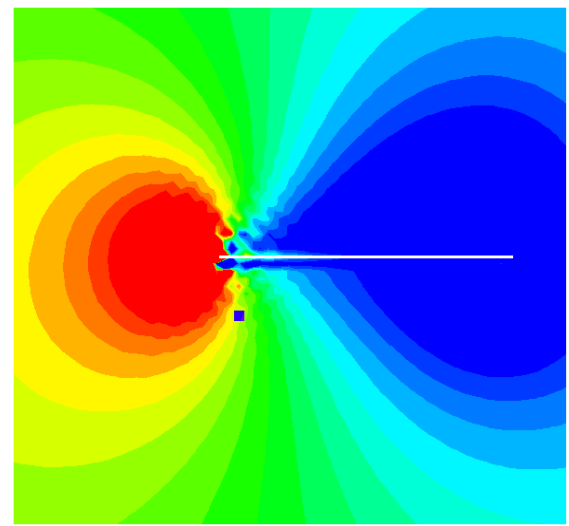

(2) After crossing

Figure 12: Contours of vorticity and pressure around the oscillating zero thickness plate at two time instants:

(1) before crossing lattice, and (2) after crossing lattice. Arrow indicates the direction of wing movement. 
C. Wing with delayed rotation hovering at $R e=100$

The complex fluid dynamics around a zero-thickness flat plate undergoing hovering motion is simulated, using different treatments of the moving boundary condition, for the case of delayed rotation of the plate. The parameters governing the kinematics were chosen to be the following: $x_{a} / c=1, f=1 \mathrm{~Hz}, \alpha_{0}=90^{\circ}, \alpha_{a}=-45^{\circ}$, and $\phi=60^{\circ}$. The aerodynamic forces computed using halfway bounceback and second-order interpolation have been compared with results from an in-house finite-volume based Navier-Stokes solver [6].

Figure 13 shows force comparison between Navier-Stokes solution [6] and LBM solutions with two different noslip boundary treatments (i.e. halfway bounceback and second order interpolation). Good agreement between the LBM and Navier-Stokes results is noticeable. In addition, results obtained through second order interpolation show a relatively better agreement with the NS solution. When compared with the halfway bounceback method, the use of second-order interpolation resulted in decreased fluctuations as observed in the time history of drag and lift coefficients. However, the use of interpolation could not completely eliminate the fluctuations in the force history. In particular, the fluctuations were prominent near the time instant corresponding to $t / T=5.4$, which corresponds to the peak lift coefficient as is shown in Figure 13(b). The pressure and vorticity contours around this time instant are shown in Figure 14 and 15 respectively. It can be observed that although vorticity around the wing is smooth and continuous, pressure shows significant discontinuity at $t / T=5.4$. The high fluctuation in the forces could hence be attributed to this acoustic phenomenon.

Streamwise vorticity contours at two different wing sections, namely at mid-span and the wing-tip, and iso $Q$ contours around the wing for the NS and LBM simulations, with two different no-slip boundary treatments, are shown in Figures 15 and 16 respectively. $Q$ is defined as

$$
Q=0.5\left(R_{i j} R_{i j}-S_{i j} S_{i j}\right)
$$

where $R_{i j}$ and $S_{i j}$ are the angular rotation and rate-of-strain tensors, respectively, and are components of the velocity gradient tensor $\left(\partial u_{i} / \partial x_{j}=R_{i j}+S_{i j}\right)$. A high value of $Q$ would suggest a higher level of coherence in the vortical flow structure. The vorticity computed using LBM shows good agreement with the NS solution. Moreover, from Figure 16, it can be observed that the far field vortical structure is more dissipated, which is likely to be due to the coarse grid topology and resolution for the NS computations, as compared to the LBM solution. The threedimensional iso- $Q$ contours predicted by LBM are shown in Figure 17. The corresponding $Q$ contours at the midspan for the three different simulations are shown in Figure 18. It can be observed that the flow predictions through the halfway bounceback show significant discontinuity in the velocity field pattern near the wing surface. However, the flow field computed using second-order interpolation shows a better prediction, especially around the wing, than that with the halfway bounceback method (see Figure 18 (b) and (c)). For the Reynolds number considered in the current study, the 3D LBM computation requires substantially less time than the Navier-Stokes computation, due to the former's non-iterative nature and the utilization of a fixed grid. 


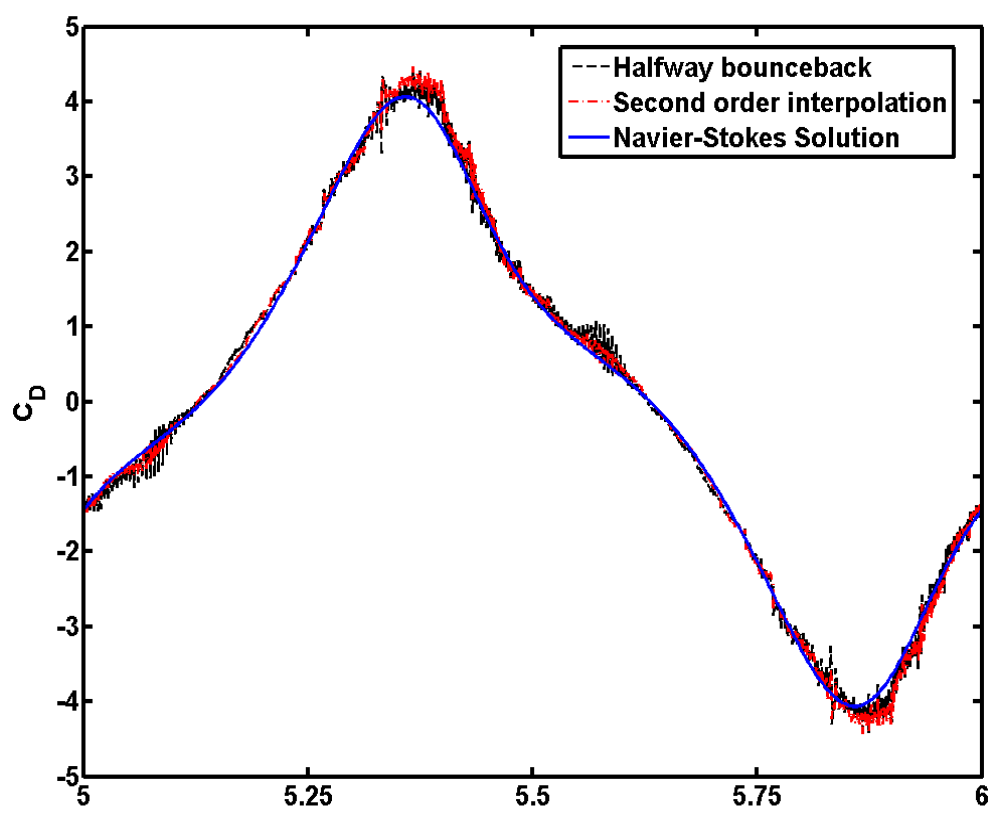

(a) Drag coefficient

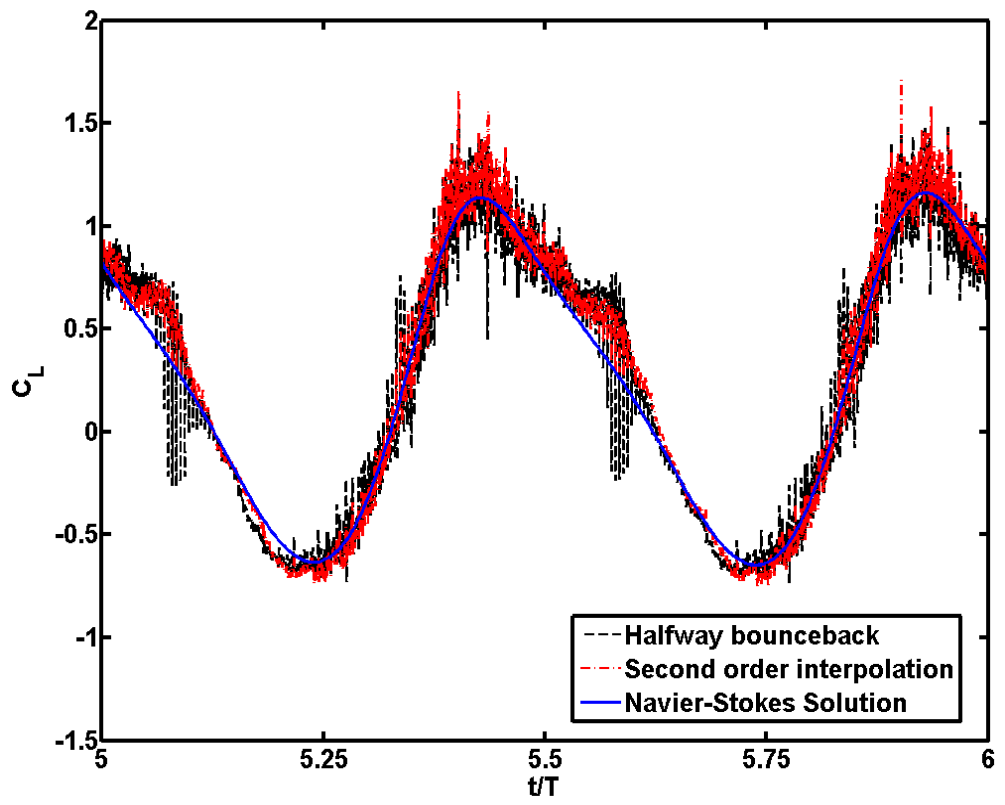

(b) Lift coefficient

Figure 13: Comparison between the results of 3D LBM simulation obtained through halfway bounceback (black dot line) and second order interpolation (red dashed line) with the Navier-Stokes simulation (solid blue line) at $\operatorname{Re}=100$ and $k=0.5$. 


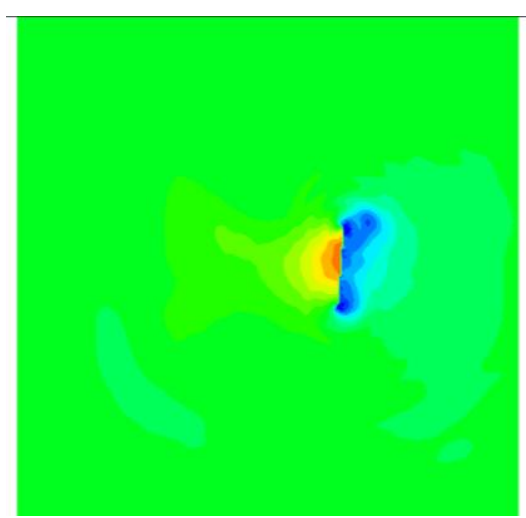

(a) $t / T=5.36$

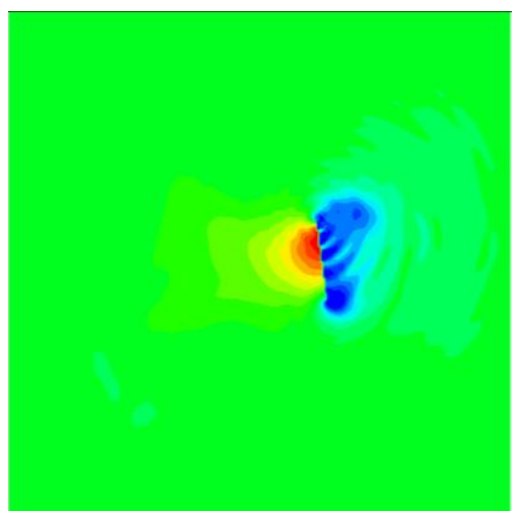

(b) $t / T=5.4$

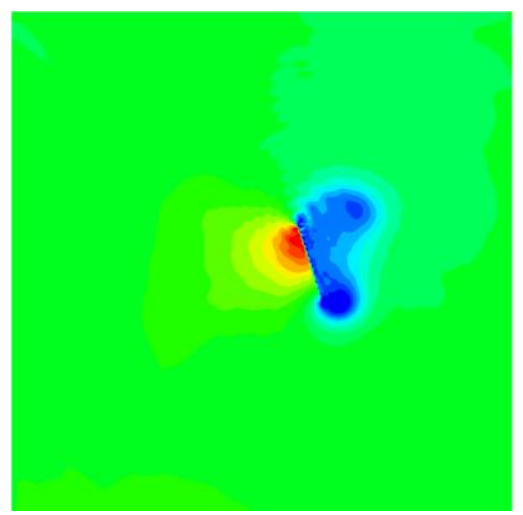

(c) $t / T=5.44$

Figure 14: Pressure contours around the hovering wing for three different time instants near the peak lift computed using the second-order interpolation at $\mathrm{Re}=100$.

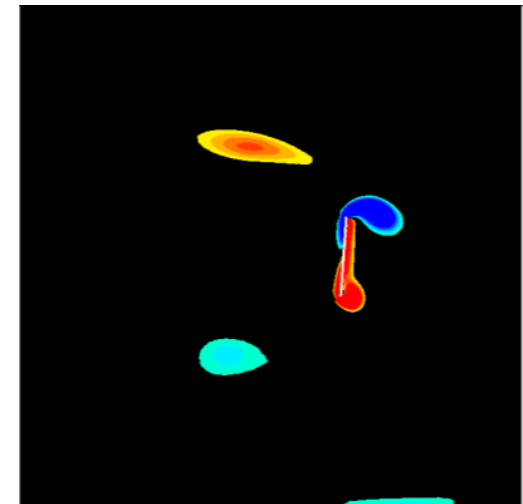

(a) $t / T=5.36$

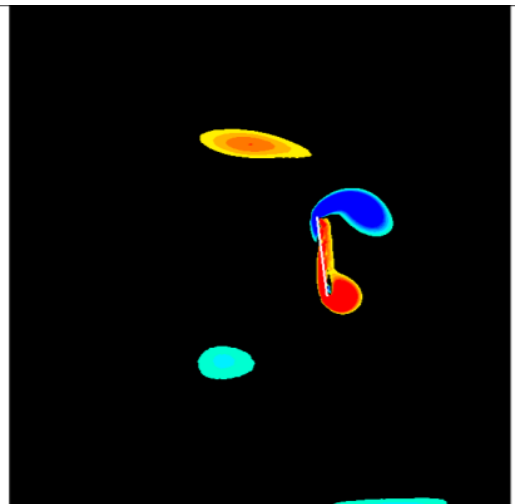

(b) $t / T=5.4$

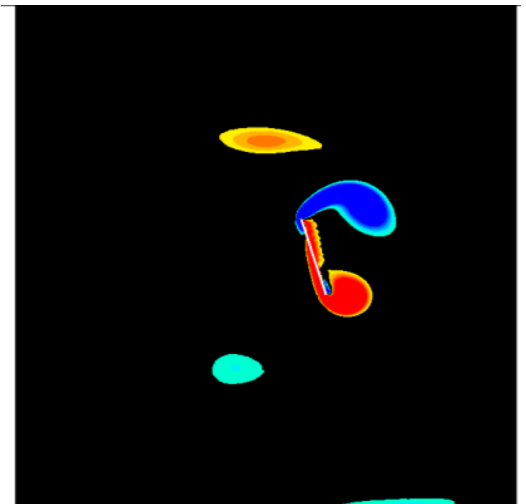

(c) $t / T=5.44$

Figure 15: Vorticity contours around the hovering wing for three different time instants near the peak lift computed using the second-order interpolation at $\mathrm{Re}=100$.

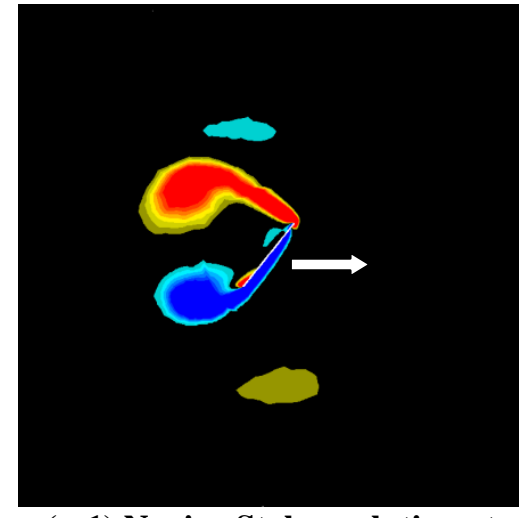

(a-1) Navier-Stokes solution at mid span

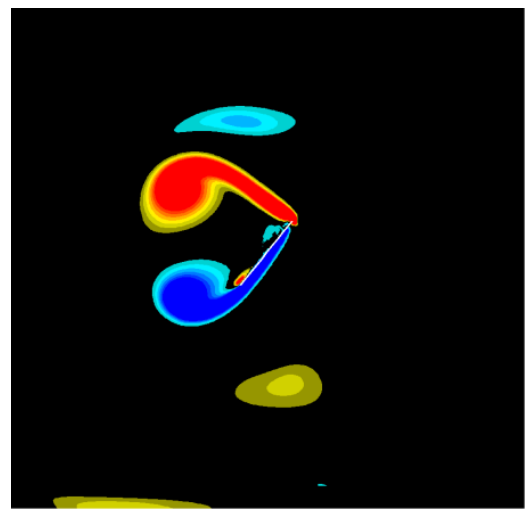

(b-1) LBM solution with halfway bounceback at mid span

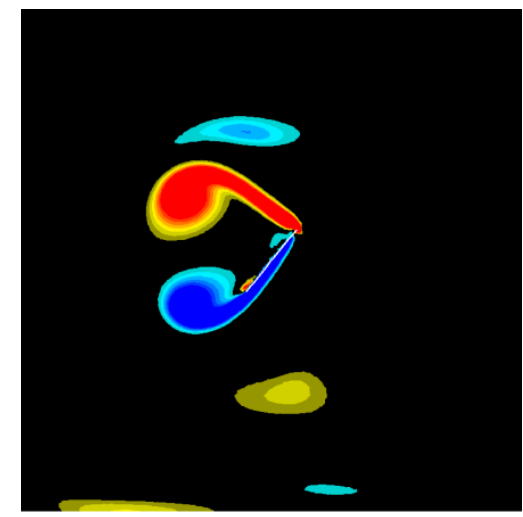

(c-1) LBM solution with second order interpolation at mid span 


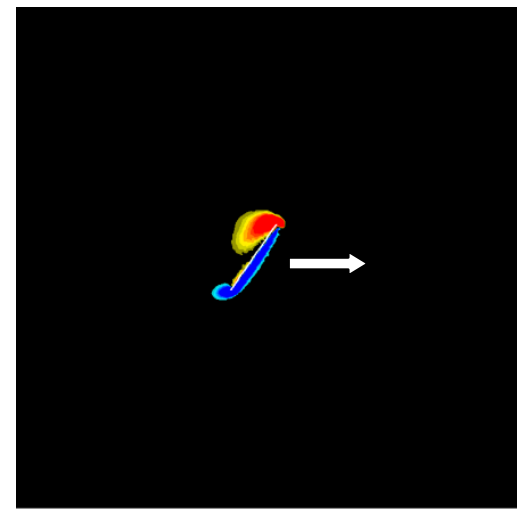

(a-2) NS solution at wing tip

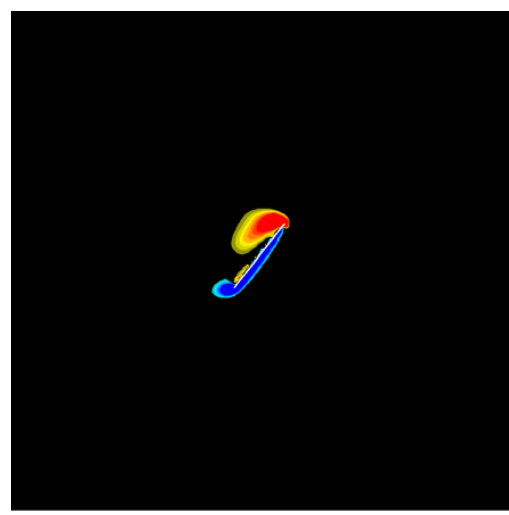

(b-2) LBM solution with halfway bounceback at wing tip

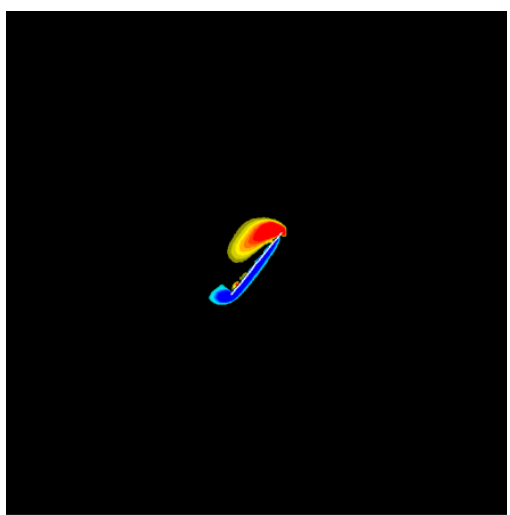

(c-2) LBM solution with second order interpolation at wing tip

Figure 16: Comparison of spanwise vorticity contours around hovering flat plate at the middle of stroke (highlighted in red in Figure 7 (a)) at $R e=100$ and $k=0.5$. Arrow indicates the translational direction of the wing movement.

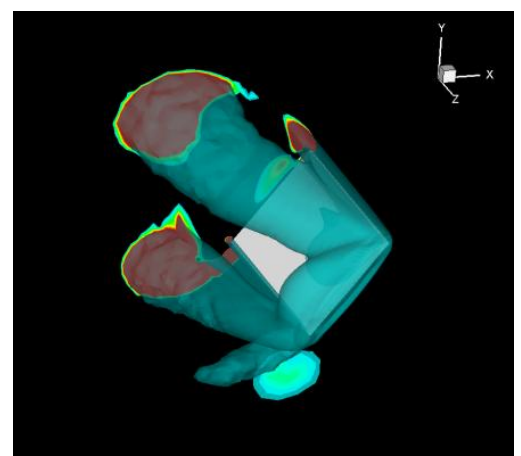

(a) NS solution

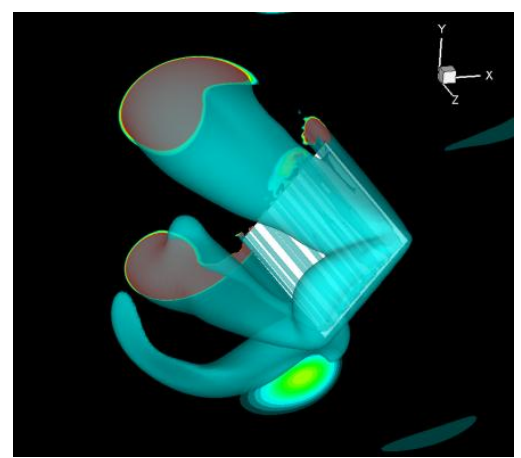

(b) LBM solution with halfway

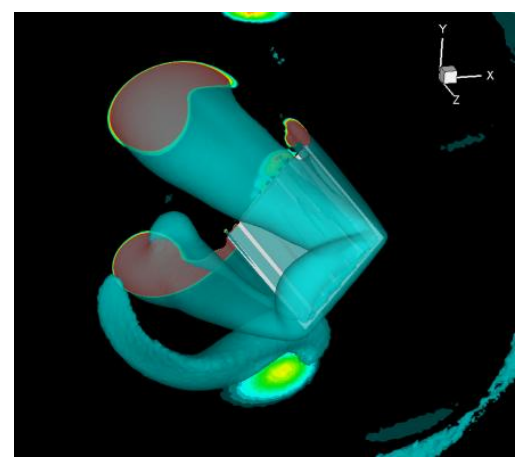

(c) LBM solution with second order interpolation

Figure 17: Comparison of iso- $Q(=4)$ surfaces snapshots around hovering flat plate at the middle of stroke (highlighted in red in Figure 7 (a)) at $R e=100$ and $k=0.5$. The wing is translating and rotating along the $X$ and $Z$-axis respectively.

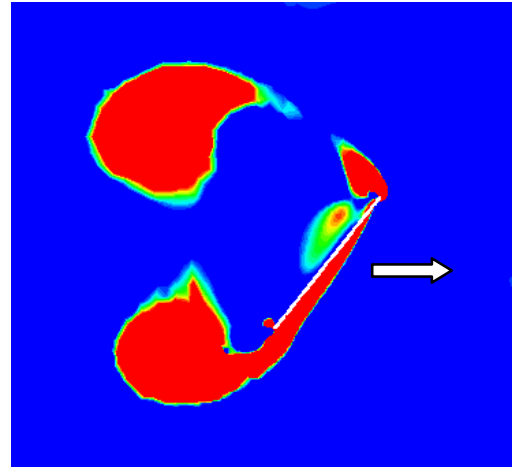

(a) NS solution at mid span

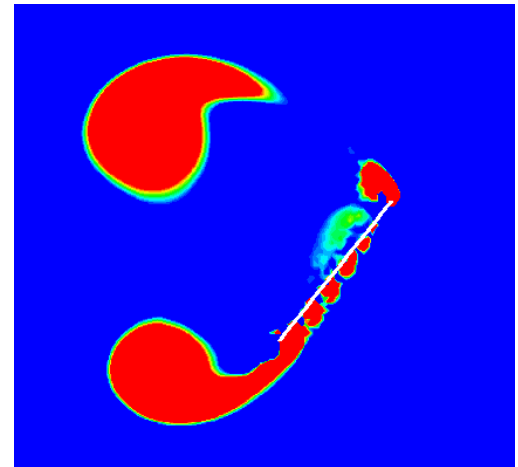

(b) LBM solution with halfway bounceback at mid span

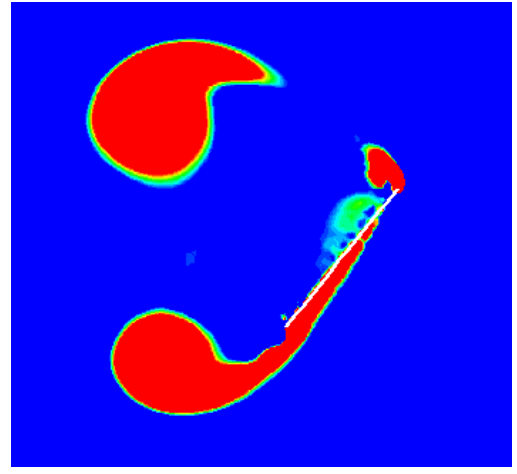

(c) LBM solution with second order interpolation at mid span

Figure 18: Comparison of iso- $Q$ contour snapshots around hovering flat plate the middle of stroke (highlighted in red in Figure 7 (a)) at $R e=100$ and $k=0.5$. Arrow indicates the direction of wing movement. 


\section{Finite thickness flat plate}

In this section, the results of two different case studies will be presented: 1) three-dimensional LBM simulation of a finite-thickness rigid wing with advanced rotation under hovering condition at $R e=136$, and 2) threedimensional LBM simulation of flexible wing with advance rotation under hovering condition at $R e=100$. The results will be compared with the available published data [6,35].

A. Wing with advanced rotation hovering at $R e=136$

Results obtained through the lattice Boltzmann simulation of a finite-thickness flat plate have been compared against a Navier-Stokes based computation [6]. The computational set-up and wing kinematics were described in sub-section D of section II. The results for lift and drag coefficients over multiple cycles, obtained through the two methods, are compared in Figure 19. It can be seen that both lift and drag coefficient computed by LBM is in good agreement with Navier-Stokes solution. The presence of two peaks in the force histories during each up- and downstroke is observable. The first peak at the beginning of a half-stroke is due to wake capture. The second peak at the end of the half-stroke is attributed to rotational circulation. These unsteady mechanisms were also found to be consistent with the earlier published findings [36].

\section{B. 3D Flexible wing simulation with advanced rotation at $R e=100$}

The computed lift forces using the present method have been compared with those using the viscous vortex particle method (VVPM) [20, 35]. The time history of the resultant angle of deflection is directly compared with those of experiments [35], and is shown in Figure 20. The 'driver' segment was prescribed the kinematics as explained in sub-section D of section II earlier. The 'driven' segment responds to the motion of the 'driver', with the links connected at all times through a ball-and-socket joint attached at their respective ends. Two different mesh spacing were simulated to test for grid independence of the presented results. As shown in Figure 20, refinement of the mesh did not lead to any appreciable change in the magnitude of the vertical force and the deflection angle. In addition, the vertical force and the deflection angle were found to be in good agreement with the experiments [35].

The pressure and vorticity contours obtained using the flexible wing model of LBM are shown in Figure 21. The wing configuration, including the bending angle between the 'driver' and 'driven' segment is also shown. From Figure 21(b), the presence of a trailing edge vortex that is attached to the 'driven' segment is observable. The presence of a low pressure region on the upper side of the two segments can be discerned as well. This contributes to the generation of a positive lift at this time instant, as is shown in Figure 20(a).

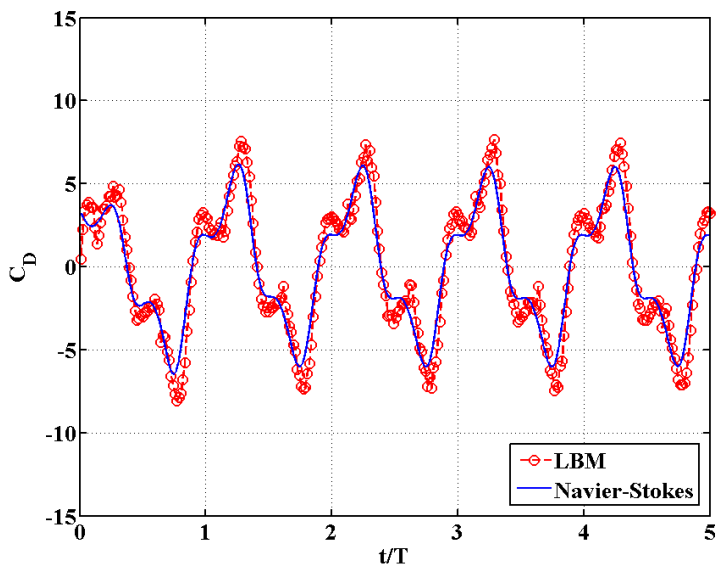

(a) Drag coefficient

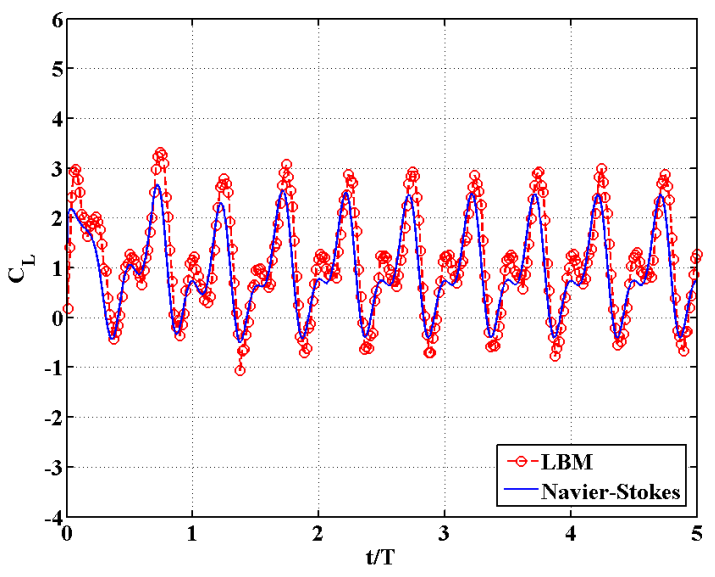

(b) Lift coefficient

Figure 19: Comparison between the results of 3D LBM and Navier-Stokes simulations at $R e=136$ and $k=0.71$ 


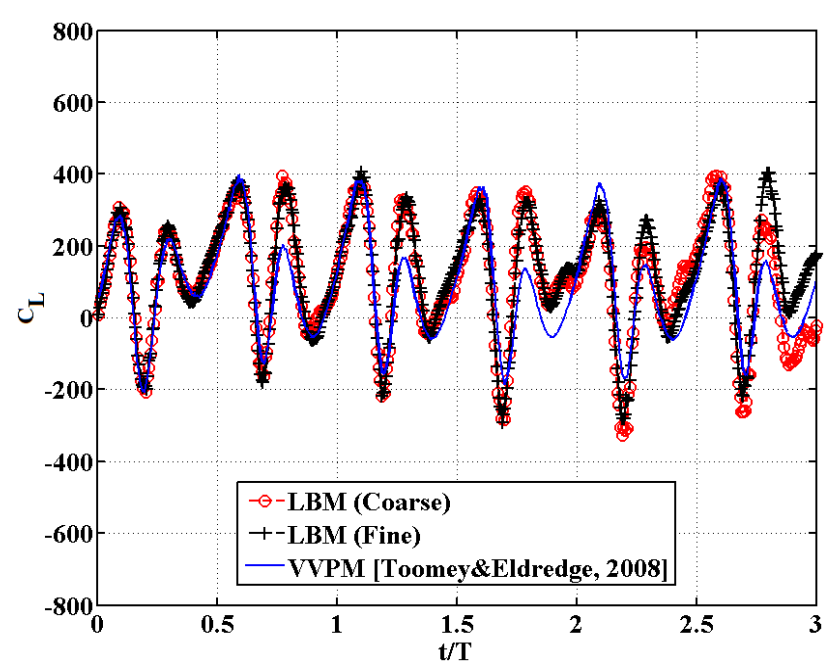

(a) Lift coefficient

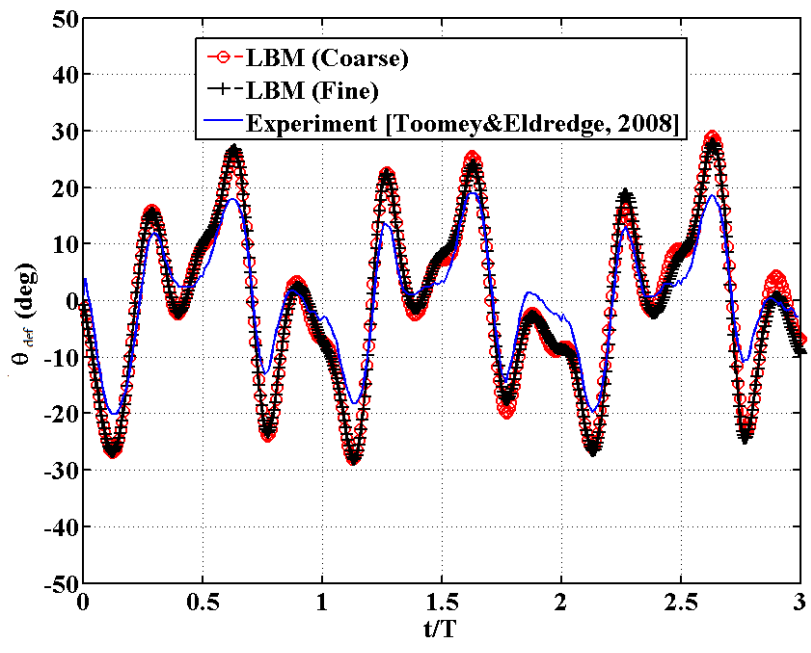

(b) Deflection angle $\left(\theta_{\text {def }}\right)$

Figure 20: Comparison between the results of 3D LBM simulation, VVPM simulation, and the experiment at $R e=100$ and $k=0.71$.

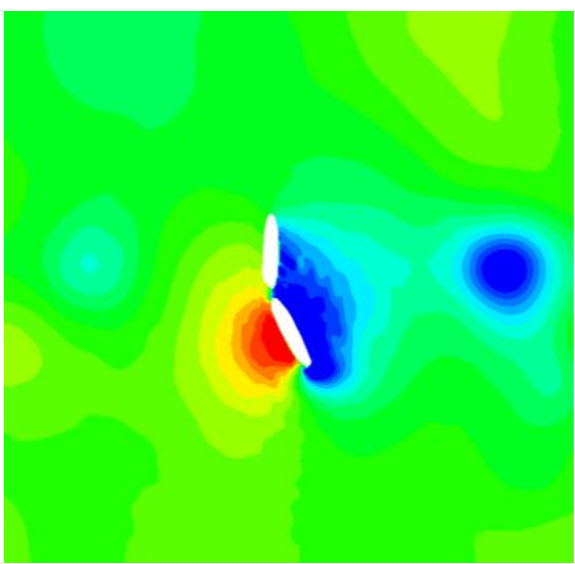

(a) Pressure

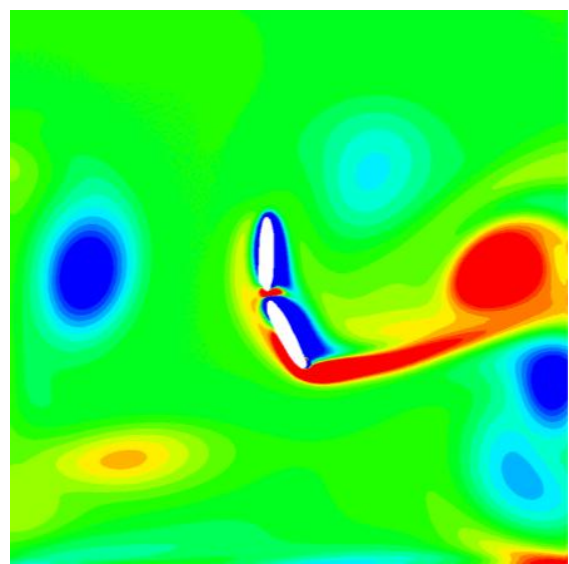

(b) Vorticity

Figure 21: Contour plots, taken at middle of span, of (a) pressure, and (b) vorticity at $R e=100$ using the 3D lattice Boltzmann flexible wing simulation. The upper and lower wings denote the 'driver' and 'driven' segments respectively. The wing is moving in the westward direction in the current configuration taken at $t / T=2.0$.

\section{Conclusions}

In this work, various treatments of the no-slip condition, namely halfway bounceback, first- and second-order interpolations, for the moving boundary problem of rigid and flexible wings flapping in a fluid at rest have been analyzed. The halfway bounceback method has a popular choice for imposing the no-slip boundary condition; however, its accuracy in resolving the true shape of thin and irregularly aligned objects can be compromised due to the staircase pattern associated with the exchange of momentum in the key step of this method. Hence, treatments based on interpolation were also considered in the scope of flapping and plunging wing simulations to analyze the accuracy of the solution near the moving boundary. Through the use of simple examples, such as uniform flow past stationary and oscillating flat plates, it was shown that the halfway bounceback, first- and second-order interpolation yield velocity profiles that were consistent with a finite-volume based Navier-Stokes solver. For the case of flow past a stationary plate, interpolation schemes unlike the halfway method, were found to resolve the exact location of the wall accurately when the distance from the nearest lattice, $\Delta \neq 1 / 2$. 
Flow past a vertically oscillating plate at $R e=100$ was used as a preliminary exercise to explain the presence of fluctuations in the aerodynamic force history. Two different amplitudes were simulated: $0.49 \delta_{x}$ and $0.51 \delta_{x}$. For the former, the lift and drag coefficients did not show any fluctuations. The latter case showed large fluctuations at the time instant when the plate crossed into the adjoining lattice. This crossing of the nodes resulted in reversal of the direction of momentum transfer from the moving boundary. For the case with the higher amplitude, momentum transfer occurs along link directions that are dynamic and change with the position of the plate. Current results suggest that the crossing of the plate and reversal of the bounce-back links is the main source of large fluctuations in the forces. Although the velocity field shows negligible change due to reversal of bounceback, the large fluctuations could be attributed to the sudden appearance of a low pressure region, and hence a large pressure differential, near the plate after it has crossed into the adjacent cell. The dissimilarity in the prediction of macroscopic variables before and after crossing of a lattice indicates different levels of accuracy of pressure and velocity around the moving boundary in LBM. Since the exhibition of lower level of accuracy in pressure has not been analyzed so far, further investigation is necessary and will be directed towards possible improvements applicable to this aspect of moving boundary simulations in the framework of LBM.

The treatment of the boundary condition, accuracy of the computed pressure and velocity fields are key to flapping wing computations. To analyze these aspects, three-dimensional simulations were conducted for a zerothickness flat plate undergoing hovering motion using different treatments of the moving boundary condition, for the case of delayed rotation of the plate. Results obtained through second order interpolation show better agreement with the NS solution, as compared to the halfway bounceback method. The use of second interpolation resulted in decreased fluctuations in the time history of drag and lift coefficients when compared with the halfway bounceback method. Moreover, the flow predictions through the halfway bounceback show significant discontinuity in the velocity field pattern near the wing surface. The flow field computed using second-order interpolation indicated a more continuous representation of the flow near the wing. In lattice Boltzmann simulations, the computational time is directly linked to the choice of the dimensionless relaxation parameter $(\tau)$; a lower value of this parameter will mean a longer time to complete one time-period of wing hovering motion. For the Reynolds number considered in the current study, with the lowest relaxation time parameter chosen $(\tau=0.59)$, employing LBM indicated significant savings in computational time as compared to the Navier-Stokes simulation, due to the former's noniterative nature and the utilization of a fixed grid.

Results obtained through the lattice Boltzmann simulation of a finite-thickness flat plate were found to be in good agreement with the Navier-Stokes solution. Preliminary results of a three-dimensional two-link flexible wing model undergoing pitching and plunging motion were also presented. It was shown that vertical force computed using the three-dimensional lattice Boltzmann-based flexible wing model is in good agreement with the experimental findings. In addition, the deflection of the passive link closely resembles the true deflection as was observed in the same experiments. In the future, simulations with the effect of span-wise flexibility using the flexible-wing model will be presented.

\section{Acknowledgments}

The work has been supported by in part by the Air Force Office of Scientific Research's Multidisciplinary University Research Initiative (MURI) and by the Michigan/AFRL (Air Force Research Laboratory)/ Boeing Collaborative Center in Aeronautical Sciences. We would like to acknowledge Dr. J. Eldredge for providing flexible flapping wing experimental data for direct comparison with the present study.

\section{References}

[1]. Shyy, W., Lian, Y., Tang, J., Viieru, D., and Liu, H. Aerodynamics of Low Reynolds Number Flyers. New York: Cambridge University Press; 2008.

[2]. Platzer, M., Jones, K., Young, J. and Lai. J., "Flapping Wing Aerodynamics: Progress and Challenges," AIAA Journal, Vol. 46, No. 9, 2008, pp.2136-49.

[3]. Muller, T. J., Fixed and Flapping Wing Aerodynamics for Micro Air Vehicle Applications. AIAA Prog Astronaut Aeronaut 2001:195.

[4]. Stanford, B.K., Ifju, P., Albertani., R., and Shyy, W., "Fixed Membrane Wings for Micro Air Vehicles: Experimental Characterization, Numerical Modeling, and Tailoring," Progress in Aerospace Sciences, Vol. 44, No. 4, 2008, pp.258-294.

[5]. Shyy, W., Aono, H., Chimakurthi, S. K., Trizila, P., Kang, C-K., Cesnik, C.E.S, and Liu, H., "Recent Progress in Flapping Wing Aerodynamics and Aeroelatcisity," Progress in Aerospace Sciences, in press. 
[6]. Shyy, W., Trizila, P., Kang, C. K., and Aono, H., "Can Tip Vortices Enhance Lift of a Flapping Wing", AIAA Journal, Vol. 47, No. 2, 2009, pp. 289-293.

[7]. Azuma, A., The Biokenetics of Flying and Swimming. $2^{\text {nd }}$ edition, Virginia: American Institute of Aeronautics and Austronautics Inc. 2006.

[8]. Dudley, R. The Biomechanics of Insect Flight: Form, Function, Evolution. New Jersey: Princeton University Press; 2002.

[9]. Biewener, A. A., Animal Locomotion. New York: Oxford University Press; 2003.

[10]. Ellington, C.P., van den Berg, C., Willmott, A. P., and Thomas, A. L. R., "Leading-edge Vortices in Insect Flight," Nature, Vol. 384, 1996, pp. 626-30.

[11]. Srygley, R. B., and Thomas, A. L. R., "Unconventional Lift-generating Mechanisms in Free-flying Butterfies," Nature, Vol. 420, 2002, pp. 660-664.

[12]. Hendenström, A., Johansson, L. C., Wolf, M., von Busse, R., Winter, Y., and Spedding, G. R., "Bat Flight Generates Complex Aerodynamic Tracks," Science, Vol. 316, 2007, pp. 894-897.

[13]. Sui, Y., Chew, Y-T., Roy, P., and Low, H-T., "A Hybrid Immersed-boundary and Multi-block Lattice Boltzmann Method for Simulating Fluid and Moving-boundary Interactions," ," Internal Journal for Numerical Methods in Fluids, Vol. 53, 2007, pp. 1727-1754.

[14]. Gao, T., Liu, N-S., and Lu, X., "Numerical Analysis of the Ground Effect on Insect Hovering," Journal of Hydrodynamics, Vol. 20, No.1, 2008, pp.17-22.

[15]. Gao, T, and Lu, X., "Insect Normal Hovering Flight in Ground Effect," Physics of Fluids Vol. 20, 2008, 087101.

[16]. Liu, Y., Liu, N., and Lu, X., "Numerical Study of Two-winged Insect Hovering Flight," Advances in Applied Mathematics and Mechanics, Vol. 1, No. 4, 2009, pp.481-509.

[17]. Weis-Fogh, T., "Quick Estimates of Flight Fitness in Hovering Animals," Journal of Experimental Biology, Vol. 59, 1973, pp. 169.

[18]. Wu, J., Shu, C., and Zhang, Y. H., "Simulation of Incompressible Viscous Flows around Moving Objects by a Varient of Immersed Boundary-lattice Boltzmann Method," Internal Journal for Numerical Methods in Fluids, Vol. 62, 2010, pp. 327-354.

[19]. Wang, Z. J., Birch, J. H., and Dickinson, M. H., "Unsteady Forces and Flows in Low Reynolds Number Hovering Flight: Two-dimensional Computations vs Robotic Wing Experiments," Journal of Experimental Biology, Vol. 207, 2004, pp. 449-460.

[20]. Eldredge, J. D., "Numerical Simulation of the Fluid Dynamics of 2D Rigid Body Motion with the Vortex Particle Method," Journal of Computational Physics, Vol. 221, 2007, pp. 626-648.

[21]. Zhang, J., and Lu, X-Y., "Aerodynamic Performance due to Forewing and Hindwing Interaction in Gliding Dragonfly Flight," Physical Review E, Vol. 80, 2009, 017302.

[22]. Yu, D., Mei, R., and Shyy, W., "A Unified Boundary Treatment in Lattice Boltzmann Method", 41st Aerospace Sciences Meeting and Exhibit, 2003, Reno, Nevada, USA.

[23]. Lallemand, P., and Luo, L. S., "Lattice Boltzmann Method for Moving Boundaries", Journal of Computational Physics, Vol. 184, 2003, pp. 406-421.

[24]. Okamoto, M., Yasuda, K., and Azuma, A., "Aerodynamic Characteristics of the Wings and Body of a Dragonfly," Journal of Experimental Biology, Vol. 199, 1996, pp. 281-294.

[25]. Yu, D., Mei, R., Luo, L. and Shyy, W., "Viscous Flow Computations with the Method of Lattice Boltzmann Equation," Progress in Aerospace Sciences, Vol. 39, 2003, pp. 329-367.

[26]. Mei, R., Luo, L-S. and Shyy, W., "An Accurate Curved Boundary Treatment in the Lattice Boltzmann Method", Journal of Computational Physics, Vol. 155, 1999, pp. 307-330.

[27]. Mei, R., Yu, D., Shyy, W. and Luo, L-S., "Force evaluation in the lattice Boltzmann method involving curved geometry", Physical Review E, Vol. 65, 2002, pp. 041203.

[28]. Ladd, A.J.C., "Numerical Simulations of Particulate Suspensions via a Discretized Boltzmann Equation. Part 1. Theoretical Foundation", Journal of Fluid Mechanics, Vol. 271, 1994, pp. 285-309.

[29]. Wu, J. and Aidun, C.K., "Simulating 3D Deformable Particle Suspensions using Lattice Boltzmann Method with Discrete External Boundary Force", International Journal for Numerical Methods in Fluids, Vol. 62, 2010, pp. 765-783.

[30]. Qi, D., "Direct Simulations of Flexible Cylindrical Fiber Suspensions in Finite Reynolds number flows", Journal of Chemical Physics, Vol. 125, 2006, pp. 114901.

[31]. Ziegler, D.P., "Boundary Conditions for Lattice Boltzmann Simulations", Journal of Statistical Physics, Vol. 71, 1993, pp. 1171-1177. 
[32]. Feng, Z.G. and Michaelides, E.F., "The Immersed Boundary-Lattice Boltzmann Method for Solving FluidParticles Interaction Problem", Journal of Computational Physics, Vol. 195, 2004, pp. 602-628.

[33]. Allen, M.P. and Tildesley, D.J., Computer Simulation of Liquids. Oxford: Clarendon Press; 2005.

[34]. Zou, Q. and He, X., "On Pressure and Velocity Boundary Conditions for the Lattice Boltzmann BGK Model", Physics of Fluids, Vol. 9, 1997, pp. 1591-1598.

[35]. Toomey, J. and Eldredge, J. D., "Numerical and Experimental Study of the Fluid Dynamics of a Flapping Wing with Low Order Flexibility", Physics of Fluids, Vol. 20, 2008, pp. 073603.

[36]. Dickinson, M. H., Lehmann, F. O., and Sane, S. P., "Wing Rotation and the Aerodynamics Basis of Insect Flight," Science, Vol. 284, 1999, pp. 1954-1960. 